\title{
熊果酸的结构修饰与生物活性研究进展
}

\author{
刘改枝* 李金金金史礼君刘萌芽蔡邦荣* \\ (河南中医药大学药学院 郑州 450046)
}

\begin{abstract}
摘要 熊果酸是从药用植物、水果和蔬菜中提取的具有多种药理活性的五环三萜类化合物. 近年来, 熊果酸的药理作 用得到了广泛的研究, 大量研究表明熊果酸具有多种药理活性, 包括抗癌、抗炎、抗氧化、抗菌、抗糖尿病等. 熊果酸 虽然具有广泛的生物学活性，但其水溶性差、生物利用度低等限制了在临床上的应用. 为了克服这些缺点，国内外研究 人员通过对熊果酸母体骨架进行化学修饰, 设计并开发了大量结构新颖、活性强的熊果酸衍生物. 对近年来合成的有 进一步研究价值的熊果酸衍生物及其生物活性研究进展进行总结, 为以熊果酸为先导化合物的药物研究提供参考.
\end{abstract}

关键词 熊果酸; 结构修饰; 抗肿瘤; 抗糖尿病; 抗炎; 抗菌

\section{Advances in the Study of Structural Modification and Biological Activities of Ursolic Acid}

\author{
Liu, Gaizhi* Li, Jinxin $\quad$ Shi, Lijun Liu, Mengya $\quad$ Cai, Bangrong* \\ (College of Pharmacy, Henan University of Chinese Medicine, Zhengzhou 450046)
}

\begin{abstract}
Ursolic acid (UA) belongs to a group of pentacyclic triterpenoids extracted from medicinal plants, fruits and vegetables. In recent years, the pharmacological action of ursolic acid has been widely studied. It has been reported that UA has a variety of pharmacological activities, including anti-cancer, anti-inflammation, anti-oxidation, anti-bacterial, anti-diabetes and so on. In spite of that UA shows the extensive therapeutic potential as a drug candidate, the poor water solubility and low bioavailability of UA limit its clinical application. To address these issue, large numbers of UA-based derivatives with enhanced activities and better physicochemical character have been developed by structural modification of its parent skeleton. In this paper, the research progress of the synthesized UA derivatives with therapeutic potential along with their biological activities is reviewed, which provides reference for further drug development using UA as a lead compound.
\end{abstract}

Keywords ursolic acid; structural modification; anti-tumor; anti-diabetic; anti-inflammatory; antiviral

熊果酸(Ursolic Acid, UA，图 1)又名乌索酸、乌苏 酸, 属乌素烷型(ursane)五环三萜类化合物，已发现存在 于上百种植物中, 其中许多植物为传统中药材, 在夹竹 桃、连尧叶、枇杷叶和白花蛇舌草中熊果酸的含量最为 丰富 ${ }^{[1]}$. 研究证明, 熊果酸及其衍生物具有多种生物学 效应，如抗肿瘤、抗炎、抗病毒、抗氧化、治疗糖尿病、 抗菌、神经保护和肝脏保护等作用 ${ }^{[2-4]}$. 熊果酸的结构改 造主要是在 C-3 位羟基、C- 28 位羧基、C-2 位氢及 $\mathrm{A}$ 环 等化学反应活性位点, 尤其是 C-3 位和 C-28 位同时修饰 的衍生物报道非常多. 其中 UA 及其衍生物的抗癌活性 引起了极大的关注, 特别是具有低毒性的优点, 其良好 的抗肿瘤活性、广泛的抗肿瘤途径已得到公认. 熊果酸
可与许多分子靶点相互作用，在许多细胞信号转导途径 中发挥重要作用, 通过抑制肿瘤细胞增殖、诱导调亡等 发挥抗肿瘤作用. 由于熊果酸水溶性差、生物利用度低 等，限制了其作为抗肿瘤药物的临床应用. 为了提高其 抗肿瘤、抗炎等活性, 改善其药代动力学性质, 科研工 作者以熊果酸为先导化合物开展了大量研究工作并取 得了丰硕的研究成果.

\section{1 熊果酸的结构修饰及抗肿瘤活性研究}

近年来, 随着人们对熊果酸抗肿瘤活性的深入研究 发现，熊果酸及其衍生物能够通过抑制肿瘤形成及细胞 毒作用、抑制肿瘤细胞增殖、诱导肿瘤细胞分化及调亡、

\footnotetext{
* Corresponding authors. E-mail: liugaizhi@126.com; cbr2018@hactcm.edu.cn Received February 19, 2021; revised March 17, 2021; published online May 13, 2021.

Project supported by the National Natural Science Foundation of China Postdoctoral Science Foundation (No. 2020M682311), the Doctor's Fund of Henan University of Medicine (No. 00104311-2019-33), and the Award for Science and Technology Tackling Key Problems Program of Henan Province. 中国博士后科学基金面上(No. 2020M682311)、河南中医药大学博士基金(No. 00104311-2019-33)、河南省科技攻关(No. 202102310514)资助项目.
} 


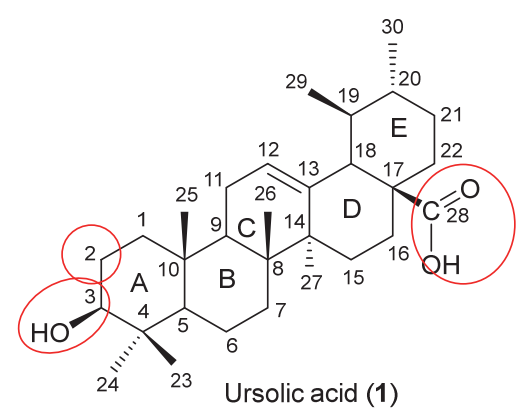

图 1 熊果酸的结构及其主要修饰位点

Figure 1 Structure of ursolic acid and its major modification sites

抗侵袭和抗肿瘤血管形成等多种途径抑制肿瘤细胞的 形成、发生和发展 ${ }^{[4-5]}$. 熊果酸的抗肿瘤研究成为近年研 究热点, 通过对熊果酸进行结构修饰已经得到了一些抗 肿瘤活性良好的衍生物, 其结构修饰主要是在 C-3 位着 基、 C-28 位羧基、 C-2 位氢、 $\mathrm{A}$ 环的修饰和改造, 许多 衍生物具有较强的抗肿瘤活性.

\subsection{C-3 位或 C-28 位的结构修饰}

只对熊果酸 C-3 位着基修饰的研究报道较少, 主要 利用其 C-3 位差基与酸反应成酯(图 2). 文献报道 C-3 位 羟基经氧化、肜化和腙化得到 3-氧代熊果酸 2、3-肜基 熊果酸 3 及 3 -苯腙熊果酸衍生物 $4^{[6]}$. 试验结果表明衍 生物 3 对肺癌 LTEP- $\alpha-2$ 细胞抑制作用最好, 优于熊果 酸, 抑制率为 $90.0 \%, \mathrm{IC}_{50}$ 为 $7.4 \mu \mathrm{mol} \cdot \mathrm{L}^{-1}$. 另外, 顺式3-O-p-差基肉桂酰熊果酸 5 是从药用植物中纯化得到的 一种 C-3 位取代熊果酸衍生物, 其通过诱导 ROS 依赖和 p53 介导的线粒体调亡, 对口腔癌细胞具有潜在的抑制
活性 ${ }^{[7]} . \mathrm{Xu}$ 等 ${ }^{[8]}$ 将分离得到的熊果酸与 3,4,5-三甲氧基 苯甲酸反应得 C-3 位含有 3,4,5-三甲氧基苯乙酰基的 UA 衍生物 6, 并在 A-549、MCF-7、H1975 和 BGC-823 四 种细胞珠中测定了其细胞毒性, UA 衍生物 6 对上述四 种细胞具有显著的抗增殖作用, $\mathrm{IC}_{50}$ 值为 $6.07 \sim 22.27$ $\mu \mathrm{mol} \cdot \mathrm{L}^{-1}$, 其中对 A549 细胞活性最佳, $\mathrm{IC}_{50}$ 值为 6.07 $\mu \mathrm{mol} \cdot \mathrm{L}^{-1}$, 且化合物 6 对正常细胞 HHDPC 无毒性. Fontana 等 ${ }^{[9]}$ 合成两个 C-3 位修饰的熊果酸衍生物 7 和 8, 在肝癌细胞株 HepG2、HA22T/VGH 和 Hep3B 上能 显著抑制细胞生长并诱导 NF- $\mathrm{NB}$ 的活化. Tu等 ${ }^{[10]}$ 合成了 系列熊果酸 C-3 位衍生物, 并评价了其对 NTUB1 细胞 的抑制活性，部分化合物表现出了良好的抑制活性，其 中化合物 9 活性最好, $\mathrm{IC}_{50}$ 值为 $(8.65 \pm 2.89) \mu \mathrm{mol} \cdot \mathrm{L}^{-1}$.

对熊果酸 C-28 位的修饰主要是与含羟基类化合物 成酯、与氨基酸形成酰胺(表 1). Gou 等 ${ }^{[11]}$ 报道熊果酸 C-28 位成酯修饰的衍生物 10 对肺癌细胞 A549 和 H460 细胞有显著的抑制作用, $\mathrm{IC}_{50}$ 值分别为 $5.4 \sim 6.1$ 和 $3.9 \sim$ $5.7 \mu \mathrm{mol} \cdot \mathrm{L}^{-1}$, 其通过诱导内质网应激诱导肺癌细胞调 亡. Tian 等 ${ }^{[12]}$ 合成了系列熊果酸 C-28 位成酯修饰的含氮 衍生物 11a 11c, 12a, 12b, 上述化合物均对 Hela 细胞 和 A549 细胞系表现出优于吉非替尼的抗增殖活性. 李 霞等 ${ }^{[13]}$ 也合成了系列熊果酸含氮衍生物，部分化合物 的抗肿瘤活性与熊果酸相比均有提高, 其中化合物 13 具有最佳的肿瘤抑制活性，对 HepG2 和 MCF-7 的抑制 活性 $\mathrm{IC}_{50}$ 值分别为 18.8 和 $19.0 \mu \mathrm{mol} \cdot \mathrm{L}^{-1}$. Mallavadhani 等 ${ }^{[14]}$ 合成了系列熊果酸 C-28 位成酯修饰的衍生物, 并 对 8 种人类癌细胞株进行抗癌活性评价, 化合物 14 17

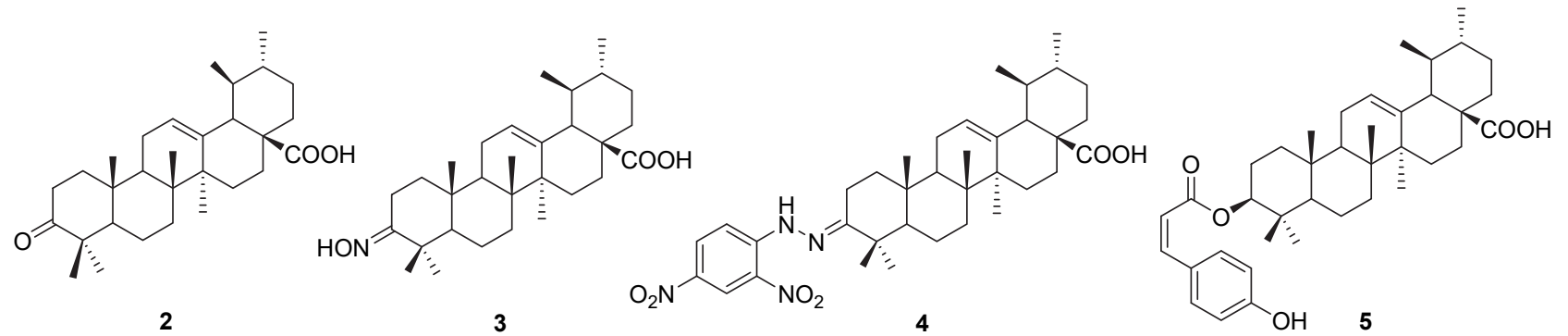

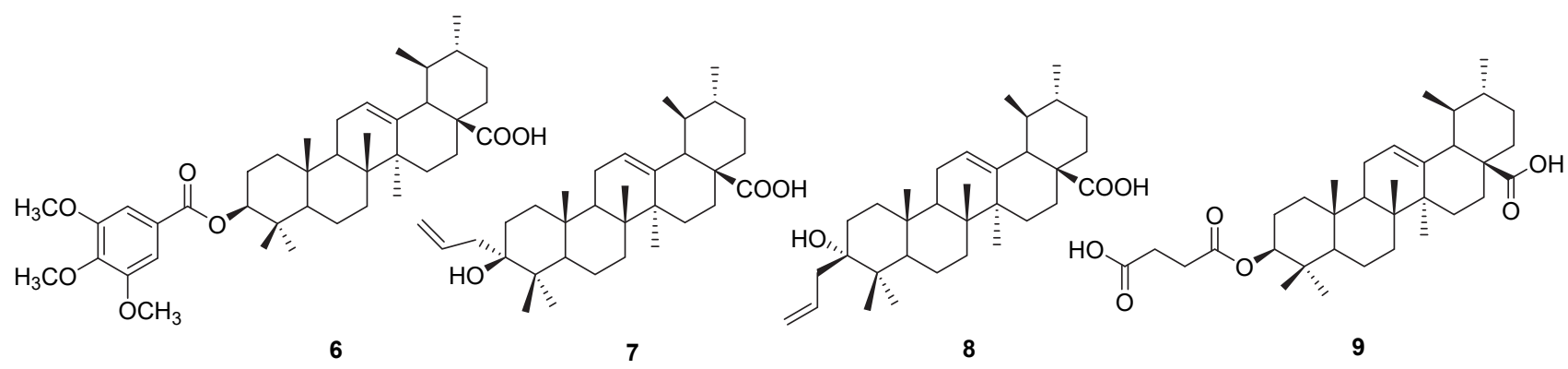

图 2 熊果酸 C-3 位修饰衍生物的结构

Figure 2 Structures of ursolic acid C-3 modified derivatives 
表 1 熊果酸 C-28 位修饰衍生物的结构

Table 1 Structures of ursolic acid derivatives modified at C-28

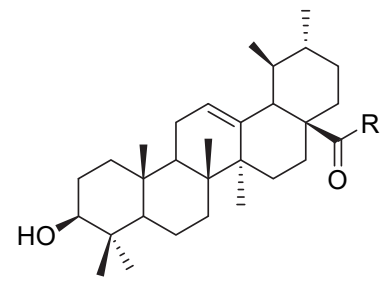

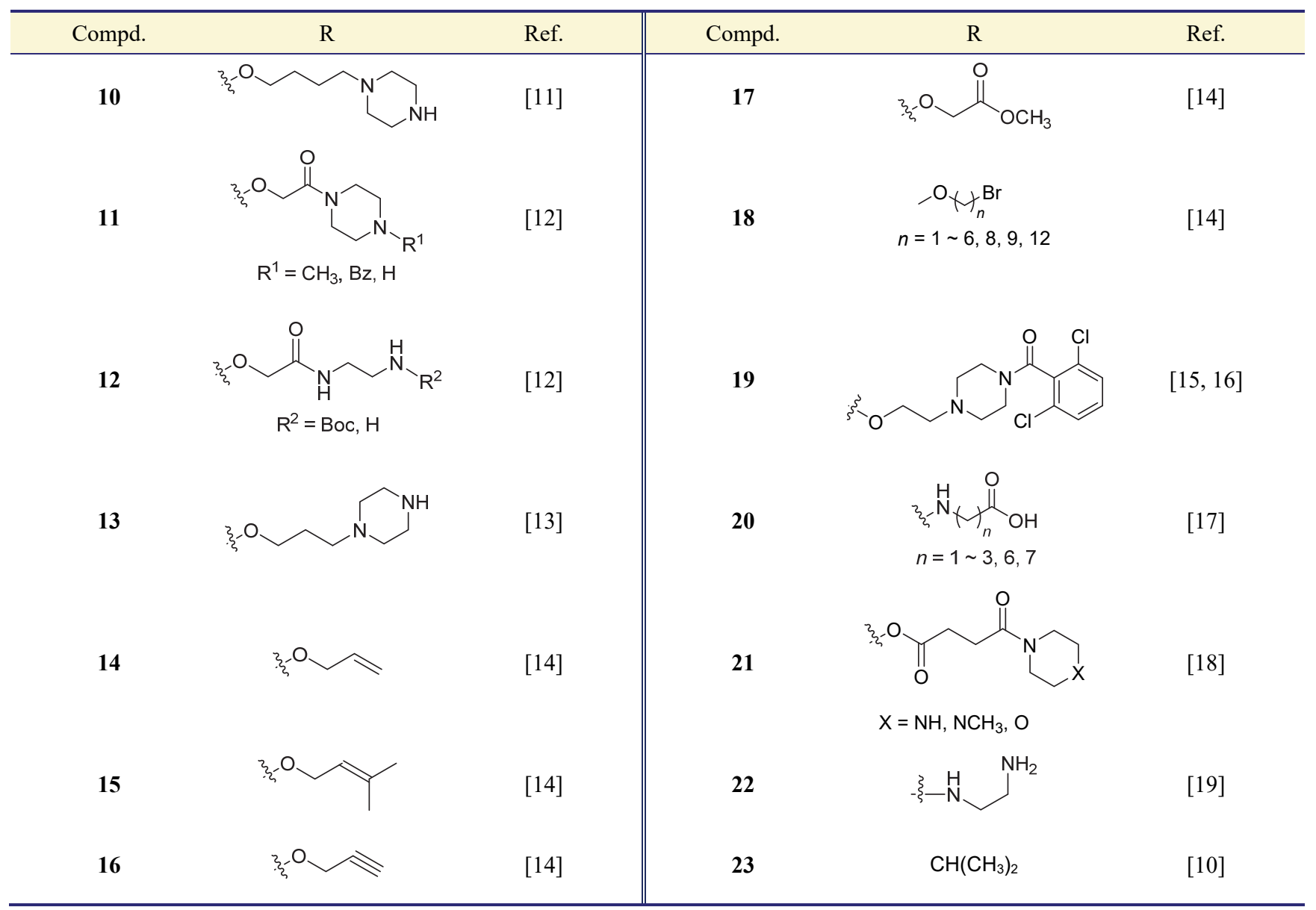

对 SiHa、HeLa、A-549 和 IMR-32 癌细胞株表现出与 UA 相当的或更好的活性. 值得注意的是, 在系列溴代 烷基酯衍生物 $18(n=1 \sim 6,8,9,12)$ 中, 化合物 $18(n=3$ 时)对白血病 THP-1 细胞株显示出最佳的抗癌活性, 构 效关系研究发现, 链长的增加对活性有不利影响. Liu 等 ${ }^{[15-16]}$ 合成了系列新型含酰基哌嗪的 UA 衍生物, 并评 价了其对 MGC-803 和 BCAP-37 人癌细胞株的体外抑制 作用. 部分化合物对被检测的癌细胞株具有中等到高水 平的抗肿瘤活性, 大多数化合物与熊果酸相比, 具有更 强的抑制活性，其中化合物 19 对 MGC-803 和 BCAP-37 两种癌细胞的抑制活性最好, $\mathrm{IC}_{50}$ 值分别是 2.50 和 9.24 $\mu \mathrm{mol} \cdot \mathrm{L}^{-1}$.

刘涵等 ${ }^{[17]}$ 合成了 $\mathrm{C}-28$ 位羧基被修饰的系列熊果酸 衍生物 $20 \mathrm{a} \sim 20 \mathrm{e}$, 构效关系研究发现, 对熊果酸 28-位
羧基进行延长修饰，有利于提高熊果酸的抗肿瘤活性， 当延长 $7 \sim 8$ 个碳原子, 抗肿瘤效果最好. 陈艳华等 ${ }^{[18]}$ 通过丁二酸连接片段, 在熊果酸 C-28 位分别导入哌嗪、 $N$-甲基哌嗪及吗啉片段，设计、合成了系列熊果酸衍生 物, 其中化合物 21b 21c ( $\mathrm{X}=\mathrm{NCH}_{3}, \mathrm{O}$ 时)对 MCF-7、 Hela 和 A549 癌细胞的抑制作用远优于 UA, 化合物 21a $(X=N H)$ 与阳性对照药吉非替尼活性相当，对 MCF-7, Hela, A549 的 $\mathrm{IC}_{50}$ 分别是 14.2, 39.01，23.42 $\mu \mathrm{mol} \cdot \mathrm{L}^{-1}$. 白锴凯等 ${ }^{[19]}$ 合成了 $\mathrm{C}-28$ 位具有电负性基团 的熊果酸衍生物 22, 并用计算软件预测衍生物油水分 配系数及水溶性的改善情况, 其水溶性相比熊果酸提高 70 倍以上, 重点研究了其对前列腺癌 PC-3 细胞的活性, $\mathrm{IC}_{50}$ 小于 $10 \mu \mathrm{mol} \cdot \mathrm{L}^{-1}$, 其抗癌机制为将细胞阻滞于 $\mathrm{G} 0 / \mathrm{G} 1$ 期并具有诱导细胞调亡作用. $\mathrm{Tu}$ 等 ${ }^{[10]}$ 也合成了系 
列熊果酸 C-28 位修饰衍生物, 部分化合物对 NTUB1 细 胞表现出了良好的抑制活性. 其中化合物 23 活性较好, $\mathrm{IC}_{50}$ 值为 $7.97 \mu \mathrm{mol} \cdot \mathrm{L}^{-1}$.

熊果酸 C-28 位修饰的衍生物还有熊果酸和低分子 肝素的偶联物 24, 可以自组装成平均粒径为 $200 \sim 250$ $\mathrm{nm}$ 的纳米胶束, 该纳米胶束在体外和体内均能抑制肿 瘤血管生成并抑制肿瘤生长 ${ }^{[20]}$. 另外叶酸-熊果酸偶联 物 25 可形成纳米粒并具有良好的肿瘤靶向性 ${ }^{[21]}$. 白锴 凯等 ${ }^{[22]}$ 合成了系列聚乙二醇 PEG 缀合熊果酸衍生物, 水溶性较熊果酸有明显改善, 同时具有更显著的体外抗 癌活性. 其中化合物 26 对 5 株受试肿瘤细胞(HepG2, BGC-823, AGS, HT-29, PC-3)的 $\mathrm{IC}_{50}$ 均小于 $10 \mu \mathrm{mol} \cdot \mathrm{L}^{-1}$ (图 3).

\subsection{C-3 位羟基、C-28 位羧基同时修饰的熊果酸衍生} 物

熊果酸 C-3 位着基和 C-28 位羧基同时修饰的衍生 物报道最多, 大多是先将 C-3 位羟基保护后 C-28 位修饰 的衍生物(如表 2 所示). 如系列 C-3 位羟基乙酰化保护 后所得到的系列 C-3 位和 C-28 位同时修饰的衍生物: Jiang 等[23]报道了熊果酸 C-28 位连有长链氨基的衍生 物, 在 A549 肺癌细胞系中对 NF- $\kappa \mathrm{B}$ 表现出非常好的抑 制活性, $\mathrm{IC}_{50}$ 值可达微摩尔级浓度, 其中化合物 27 对多 种试验肿瘤细胞系(包括多药耐药的人肿瘤细胞系)具有 较强的抑制作用, $\mathrm{IC}_{50}$ 值为 $5.22 \sim 8.95 \mu \mathrm{mol} \cdot \mathrm{L}^{-1}$, 且能成 功抑制 A549 细胞的迁移. 于舟等 ${ }^{[24]}$ 通过对熊果酸 C-3 位乙酰化和 C-28 位修饰制备了熊果酸衍生物 28, 其对 不同分化程度的人胃癌细胞株 AGS、BGC、MKN45 的
生长均有较强的抑制作用，尤其对 MKN45 细胞的生长 抑制作用较 UA 提高了近 3.5 倍. 化合物 28 能将 MKN45 细胞阻断在 $\mathrm{G} 0 / \mathrm{G} 1$ 期, 并显著诱导细胞调亡, 其作用强 度呈药物浓度依赖性. Dong 等 ${ }^{[25]}$ 通过计算机辅助药物 设计的方法，设计并合成了熊果酸衍生物 29, 其与糖酵 解抑制剂 2-脱氧- $D$-葡萄糖(2-DG)在抑制癌细胞葡萄糖 代谢中具有协同作用，该组合还具有明显的抗肿瘤活 性，且对正常细胞无明显毒性. Hua 等[26]设计合成了系 列 C-3 和 C-28 位修饰的新型熊果酸衍生物, 衍生物 30 对癌细胞抑制活性最好, 对 HepG2 细胞的 $\mathrm{IC}_{50}$ 值为 (5.40土0.79) $\mu \mathrm{mol} \cdot \mathrm{L}^{-1}$, 对 $\mathrm{MGC}-803$ 细胞的 $\mathrm{IC}_{50}$ 值为 $(9.82 \pm 0.29) \mu \mathrm{mol} \cdot \mathrm{L}^{-1}$. Huang 等 ${ }^{[27]}$ 合成了一系列含功能 化苯胺或酰胺侧链的熊果酸衍生物, 该系列化合物具有 较好的 NF- $\mathrm{KB}$ 抑制活性, 抑瘤作用评价显示 C-28 位的 功能化酰胺部分和 C-3 位的酯化对提高活性很重要, 其 中化合物 31 对肿瘤细胞的抑制效果最好，对 MGC-803、 HepG2、NCI-H460、A549 和 T24 癌细胞的 $\mathrm{IC}_{50}$ 值分别 为 $7.96 、 8.14 、 3.08 、 4.32$ 和 $5.51 \mu \mathrm{mol} \cdot \mathrm{L}^{-1}$. Wang 等 ${ }^{[28]}$ 合成了系列熊果酸 C-3 和/或 C-28 位修饰的衍生物, 其 中化合物 32 与 2-DG 联合, 通过诱导细胞调亡和糖酵解 的双重作用，对肝癌细胞增殖产生协同抑制作用. Wiemann 等 ${ }^{[29]}$ 合成了靶向癌细胞的含有羟肜类官能团 的 UA 衍生物, 部分化合物 $(33 \mathbf{a}, 33 \mathbf{b})$ 显示出良好的抗肿 瘤活性, 但对肿瘤细胞的选择性较低. Yang 等 ${ }^{[30]}$ 合成了 系列具有潜在抗癌活性的 UA 衍生物, 大多数衍生物对 肿瘤细胞的生长抑制作用强于 UA，其中化合物 34 的活 性最强, 对 12 种不同的肿瘤细胞系的 $\mathrm{IC}_{50}$ 值范围为

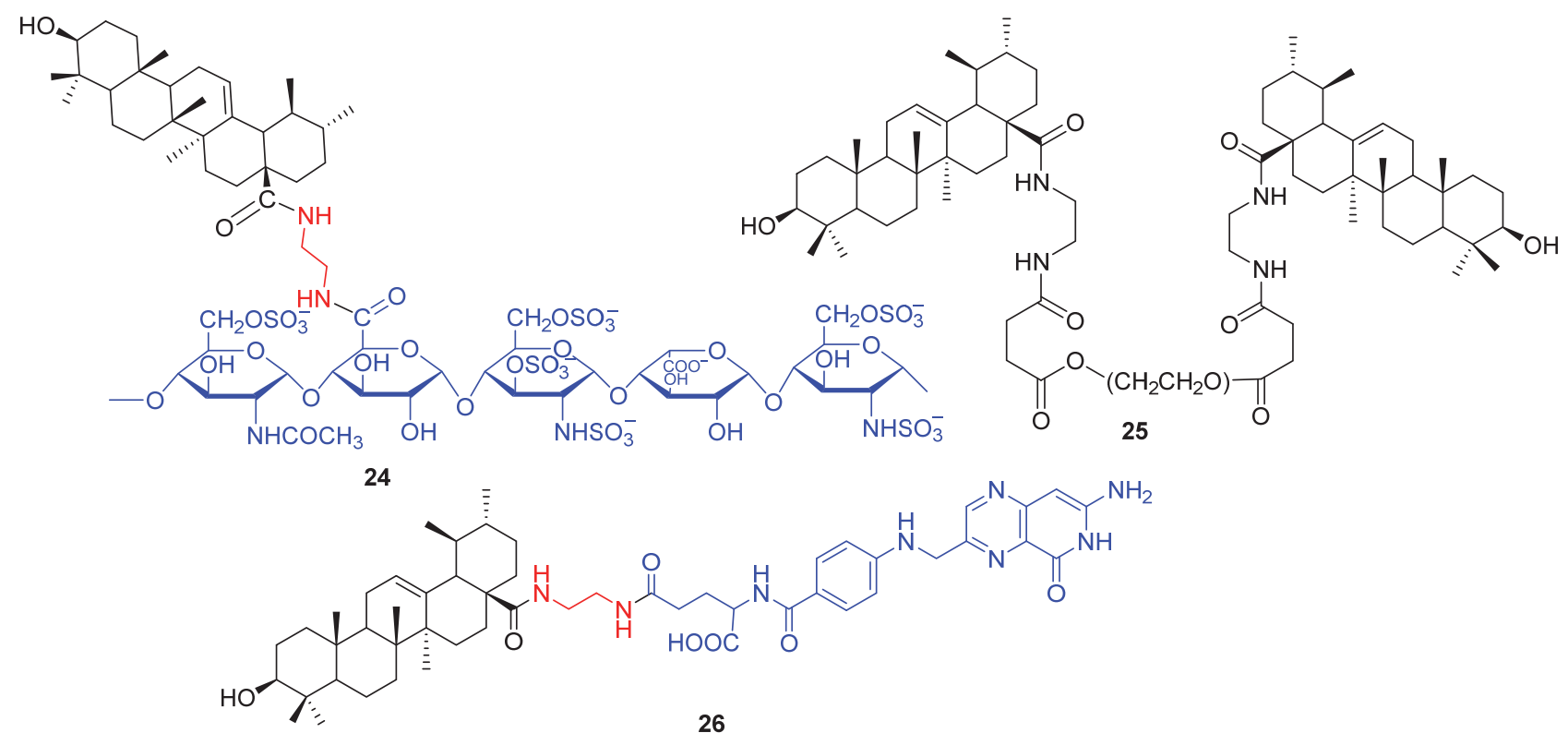

图 3 部分 C-28 位修饰熊果酸衍生物的结构

Figure 3 Structures of ursolic acid derivatives modified at C-28 
表 2 熊果酸 C-3, C-28 位同时修饰衍生物的结构

Table 2 Simultaneous modification of C-3, C-28 sites of ursolic acid derivatives

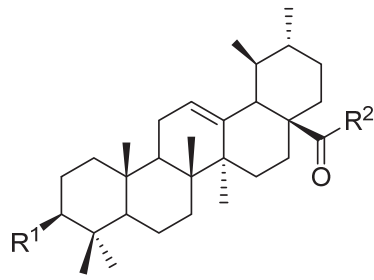

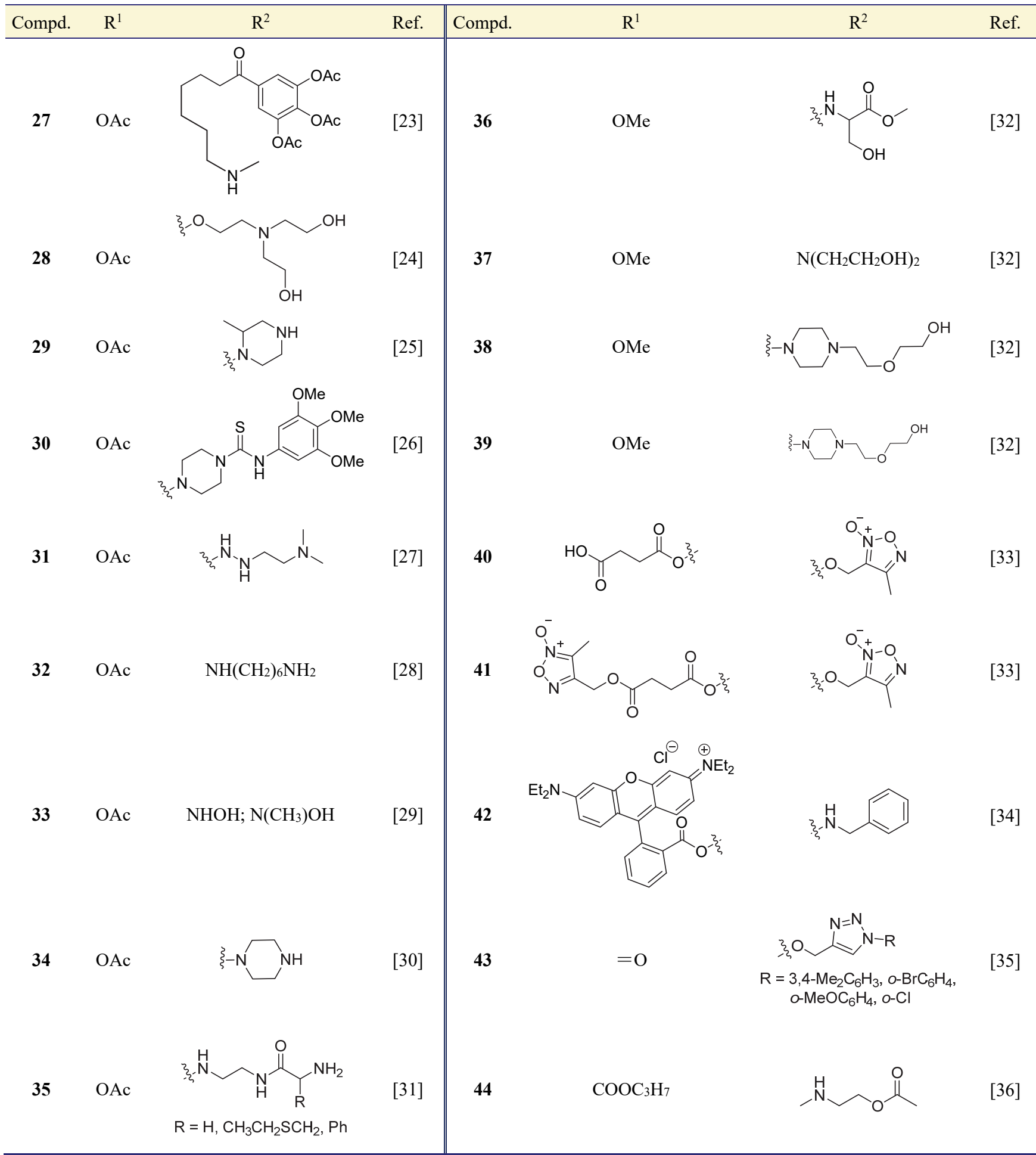


$(4.09 \pm 0.27) \sim(7.78 \pm 0.43) \mu \mathrm{mol} \cdot \mathrm{L}^{-1}$, 表现出很强的广 谱抗癌能力. 白锴凯等 ${ }^{[31]}$ 合成了系列含有伯氨基的氨 基酸修饰 UA 衍生物 35, 并评价了其对胃癌细胞株 BGC-823、 $\mathrm{AGS}$ 的体外抑制活性, 大部分衍生物对 BGC-823、AGS 的抑制活性强于 UA, 其作用机制为诱 导细胞凋亡.

Shao 等 ${ }^{[32]}$ 合成了系列 C-3 位甲氧基保护和 C-28 位 修饰的 UA 衍生物, 其中化合物 36 39 对 HepG2 细胞 的抑制效果较好, 选择化合物 38 研究其作用机制发现, 可呈剂量依赖性诱导 HepG2 细胞调亡, 并在 S 期出现细 胞周期停滞.

Popov 等 ${ }^{[33]}$ 设计合成了含 $\mathrm{NO}$ 供体的呋喃氧烷基的 熊果酸缀合物 40 和 41 , 其细胞毒性活性超母体药物熊 果酸和阳性对照药 5-氟尿嘧啶. Wolfram 等 ${ }^{[34]}$ 合成了熊 果酸 C-28 位甲酯、茮酯和茮胺的系列衍生物, 并在此基 础上进一步合成了 C-3 位与罗丹明 B 酯化的衍生物 42, 其对系列人癌细胞的抑制活性最强, $\mathrm{EC}_{50}(\mathrm{SRB})$ 为 A2780: $34 \mathrm{nmol} \cdot \mathrm{L}^{-1}, \mathrm{MCF}-7$ : $30 \mathrm{nmol} \cdot \mathrm{L}^{-1}, \mathrm{SW} 1736: 24$ $\mathrm{nmol} \cdot \mathrm{L}^{-1}$, HT29: $45 \mathrm{nmol} \cdot \mathrm{L}^{-1}$. Rashid 等 ${ }^{[35]}$ 合成了系列熊 果酸三唑啉类抗癌衍生物, 对所合成化合物对 A-549、

MCF-7、HCT-116、THP-1 和正常人上皮细胞系(FR-2) 四种人类癌细胞株进行了抗肿瘤活性评估. 与 UA 相比, 大多数化合物具有较高的抗肿瘤活性. 其中化合物 43a 43d 活性最好, 对部分癌细胞的 $\mathrm{IC}_{50}$ 值小于 0.1 $\mu \mathrm{mol} \cdot \mathrm{L}^{-1}$.

孟艳秋课题组 ${ }^{[36-39]}$ 为了研究熊果酸抗肿瘤的构效 关系, 设计合成了系列 C-3 位和 C-28 位同时修饰的熊果 酸衍生物(图 4), UA 衍生物 44 和 $\mathbf{4 5}^{[36]}$ 对 A549 和 SGC-7901 细胞的抑制效果高于阳性对照药氟尿嘧啶和
吉非替尼，化合物 46 48 对两种细胞株 BEL7402 和 SGC7901 的抑制率均明显高于母体化合物, 其 $\mathrm{IC}_{50}$ 与阳 性对照药阿霉素的 $\mathrm{IC}_{50}$ 值相当或更强 ${ }^{[40]}$. 该课题组 ${ }^{[41]}$ 还设计合成了系列乌苏烷型-2,12-烯-28-羧酸酯类熊果 酸衍生物，其中化合物 49 对 A549 肿瘤细胞的抑制率 略低于阳性对照药吉非替尼，化合物 50 ${ }^{[42]}$ 对 SKOV3 细 胞的抑制活性均明显强于熊果酸. 该课题组 ${ }^{[43]}$ 还设计 合成了 C-2 位氢、 C-3 位羟基和 C-28 位羧基以及 $\mathrm{A}$ 环 同时进行结构改造的衍生物，设计并合成了 8 个新的熊 果酸衍生物. 该类化合物对 HeLa、HepG2 和 BGC-823 细胞增殖具有一定程度的抑制作用，其中化合物物 51 和 52 对 HeLa、HepG2 和 BGC-823 细胞表现出很强的 抑制活性，并且该类化合物对于上述细胞的抑制作用明 显高于已上市药物吉非替尼.

\section{$1.3 \mathrm{~A}$ 环及其他位点修饰的熊果酸衍生物}

许多关于熊果酸 A 环修饰或 A 环结合 C-28 位同时 修饰的衍生物的设计、合成及生物活性研究已被报道, 并篮选出许多优异的候选化合物(图 5).

$\mathrm{Li}$ 等[44]设计合成了系列熊果酸 $\mathrm{A}$ 环修饰的吲哚衍 生物, 具有显著的抗癌活性, 其中化合物 53 对癌细胞株 SMMC-7721 和 $\mathrm{HepG} 2$ 的 $\mathrm{IC}_{50}$ 值分别为( $\left.0.56 \pm 0.08\right)$ 和 $(0.91 \pm 0.13) \mu \mathrm{mol} \cdot \mathrm{L}^{-1}$, 对人正常肝细胞系 $\mathrm{LO} 2\left[\mathrm{IC}_{50}=\right.$ (10.58 \pm 0.52$\left.) \mu \mathrm{mol} \cdot \mathrm{L}^{-1}\right]$ 的细胞毒性显著降低. Jin 等 ${ }^{[45]}$ 设计、合成并篎选了一系列具有酰肼、啞二唑或噻二唑 基的熊果酸喹啉衍生物, 其中化合物 54 对三种癌细胞 株(MDA-MB-231、HeLa 和 SMMC-7721)表现出最强的 抗增殖活性 $\left[\mathrm{IC}_{50}\right.$ : $(0.12 \pm 0.01),(0.08 \pm 0.01)$ 和 $0.34 \pm$ $\left.0.03 \mu \mathrm{mol} \cdot \mathrm{L}^{-1}\right]$, 强于阳性对照依托泊苷. 刘新宇等 ${ }^{[46]}$ 在
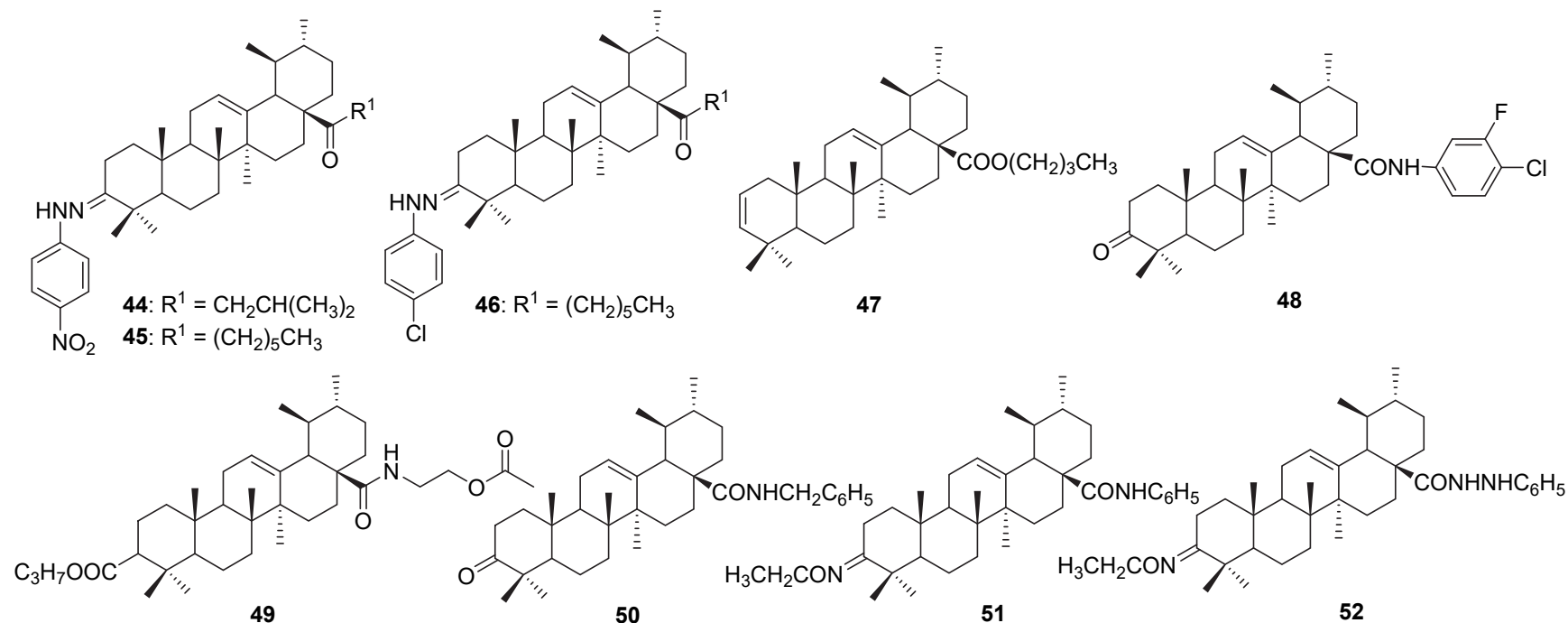

图 4 孟艳秋课题组合成的部分 C-3 位和 C-28 位同时修饰的熊果酸衍生物结构

Figure 4 Structures of ursolic acid derivatives modified simultaneously at C-3 and C-28 sites by Meng yanqiu group 

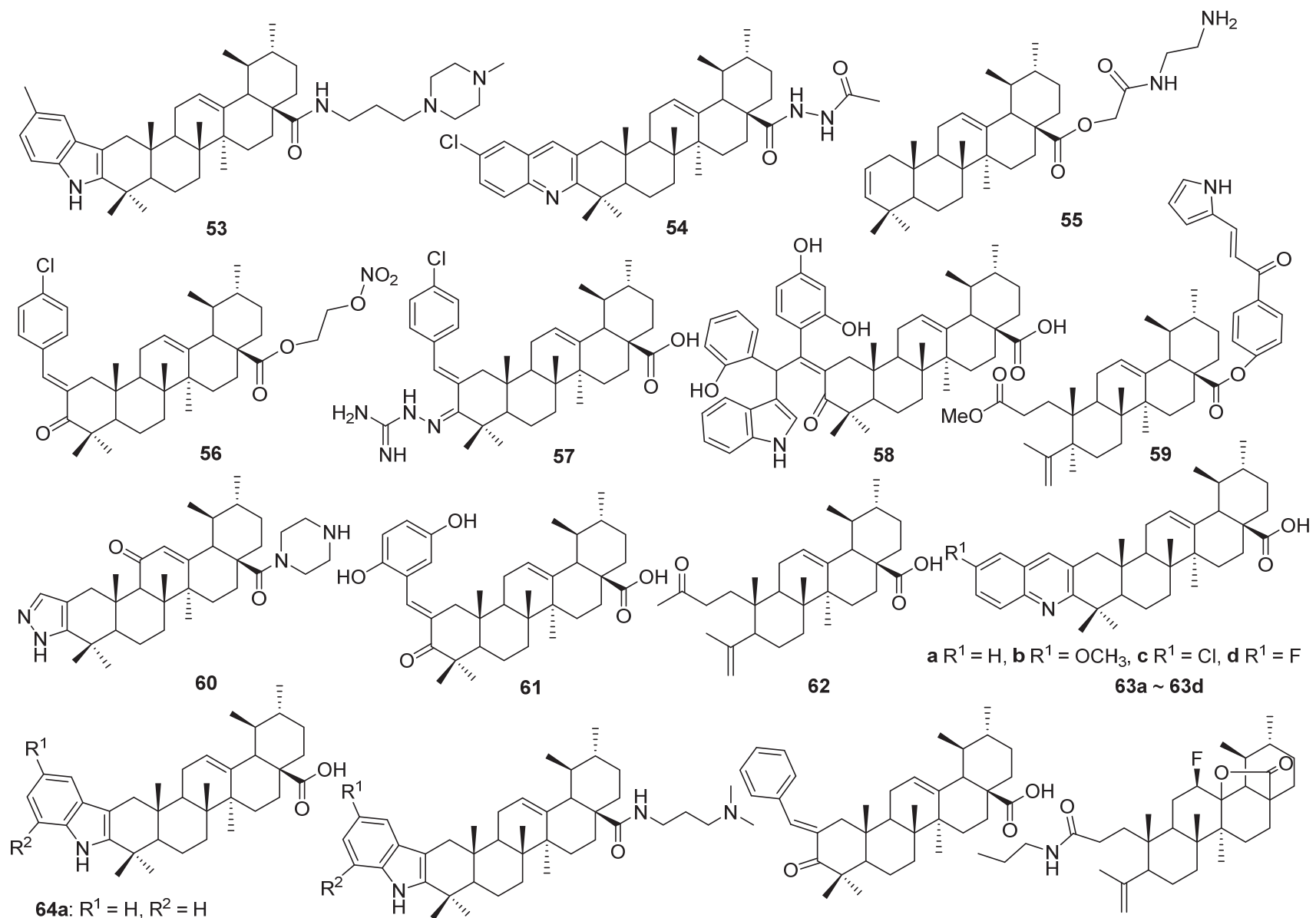

64a: $R^{1}=H, R^{2}=H$

64b: $R^{1}=F, R^{2}=H$

65a: $\mathrm{R}^{1}=\mathrm{CH}_{3}, \mathrm{R}^{2}=\mathrm{H}$

66

67

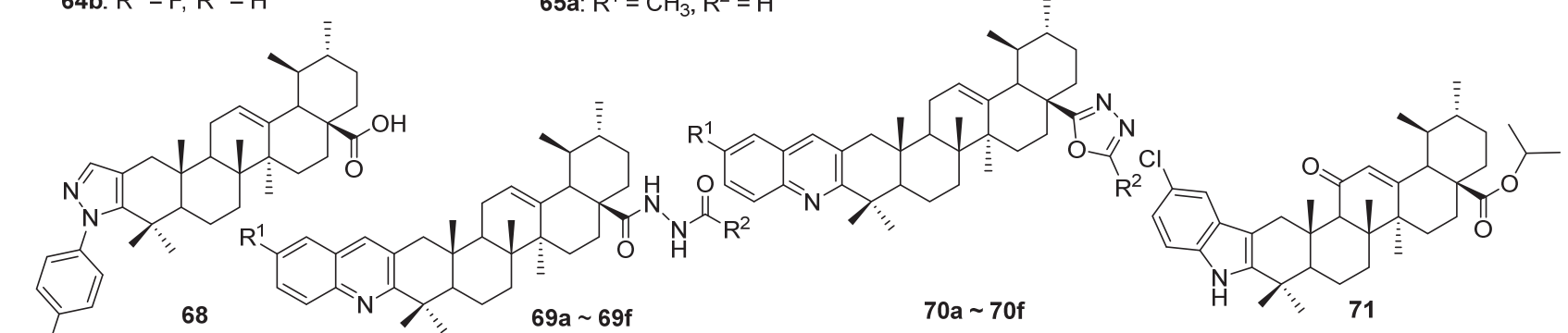

$\mathrm{CN}$

a, $\mathrm{R}^{1}=\mathrm{H}, \mathrm{R}^{2}=\mathrm{CH}_{3} ; \mathbf{b}, \mathrm{R}^{1}=\mathrm{OCH}_{3}, \mathrm{R}^{2}=\mathrm{CH}_{3} ; \mathbf{c}, \mathrm{R}^{1}=\mathrm{F}, \mathrm{R}^{2}=\mathrm{CH}_{3}$

d, $\mathrm{R}^{1}=\mathrm{H}, \mathrm{R}^{2}=\leadsto ; \mathbf{e}, \mathrm{R}^{1}=\mathrm{OCH}_{3}, \mathrm{R}^{2}=\leadsto ; \mathbf{f}, \mathrm{R}^{1}=\mathrm{F}, \mathrm{R}^{2}=\longrightarrow$

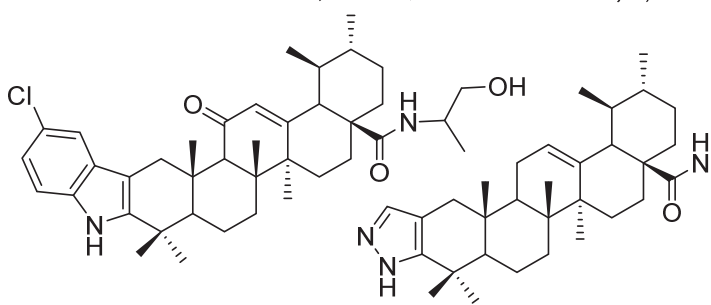

72

73

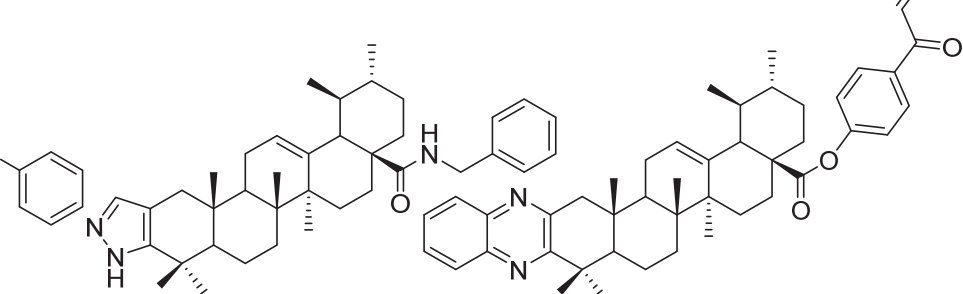

$74 \quad \mathrm{Ar}=75 \mathrm{a}: 3,4-(\mathrm{MeO})_{2} \mathrm{C}_{6} \mathrm{H}_{3}, 75 \mathrm{~b}: \mathrm{o}-\mathrm{ClC}_{6} \mathrm{H}_{4}, 75 \mathrm{c}: 2$-Furyl

图 5 A 环及其他位点修饰的熊果酸衍生物的结构

Figure 5 Structures of ursolic acid derivatives modified by A ring and other sites

熊果酸 C-28 位分别导入戊二酸和乙醇酸连接片段, 然

后与吗啉、 $N$-甲基哌嗪及 $N$-Boc 乙二胺进行偶联, 得到 系列 UA 衍生物. 其中衍生物 55 对 A549、 MCF-7 和
HepG2 三种人类癌症细胞株的活性明显优于先导化合 物 UA.

Zhang 等[47]报道了系列 NO 供体熊果酸-苄基胍衍 
生物的合成及体外抗肿瘤活性研究，其中化合物 $\mathbf{5 6}$ 对 4 株人癌细胞株(Hep G-2、MCF-7, HT-29 和 A549)都具有 良好的抑制活性，对 HT-29 最有效， $\mathrm{IC}_{50}$ 值为 4.28 $\mu \mathrm{mol} \cdot \mathrm{L}^{-1}$. $\mathrm{Wu}$ 等 ${ }^{[48]}$ 设计合成了三种新型的含胍基的熊 果酸衍生物, 并利用人癌细胞系对其进行了评价. 所合 成的熊果酸衍生物对 HIF- $1 \alpha$ 转录活性有明显的抑制作 用, 同时具有明显的体外抗癌作用, 其中化合物 57 在低 氧条件下对 HIF-1 $\alpha$ 表达的抑制效果显著, $\mathrm{IC}_{50}$ 可达到 $4.0 \mu \mathrm{mol} \cdot \mathrm{L}^{-1}$. Fan 等 ${ }^{[49]}$ 合成了系列抗胶质瘤细胞的熊果 酸杂化物, 大部分化合物对胶质瘤细胞的增殖抑制作用 优于母体熊果酸, 最有效的化合物 58 通过代谢途径下 调抑制胶质瘤细胞生长、诱导细胞凋亡和阻滞细胞周期, 其对 U251 细胞有明显的微线毛抑制和细胞表面张力增 强作用. 裴婷等 ${ }^{[50]}$ 将查尔酮通过酯化反应拼接到熊果 酸结构中, 得到系列熊果酸一查尔酮缀合衍生物, 所合 成的缀合物对 CNE2、KB、MCF-7、A549 和 HepG2 细 胞均有抑制活性, 特别是对 MCF-7 细胞的抑制活性强 于熊果酸和临床上应用的药物他莫昔芬, 其中化合物 59 的抗乳腺癌活性最强 $\left(\mathrm{IC}_{50}=4.7 \mu \mathrm{mol} \cdot \mathrm{L}^{-1}\right)$, 是他莫昔 芬 $\left(\mathrm{IC}_{50}=15.2 \mu \mathrm{mol} \cdot \mathrm{L}^{-1}\right)$ 的近 3 倍; 同时, 这些缀合物对 正常的 MCF-10A 和 VERO 细胞没有毒性, 安全性较他 莫昔芬高.

Chen 等 ${ }^{[51]}$ 设计合成了系列不同的 UA 双亲化合物， 其中两亲性化合物 60 具有显著的抗增殖作用, 并且能 在水中自组装成稳定的纳米粒子, 有望进一步应用. $\mathrm{Li}$ 等 ${ }^{[52]}$ 报道了一种熊果酸 $\mathrm{A}$ 环含氮杂环和 C-28 位双重修 饰的新型 $\mathrm{UA}$ 衍生物 60 , 研究其对 $\mathrm{BC}$ 细胞调亡诱导、 细胞增殖和细胞周期的作用发现, 化合物 60 以剂量依 赖性显著抑制乳腺癌(BC) SUM149PT 和 HCC1937 细胞 的增殖, $\mathrm{IC}_{50}$ 值为 $4 \sim 6 \mu \mathrm{mol} \cdot \mathrm{L}^{-1}$. Dar 等 ${ }^{[53]}$ 利用熊果酸与 各种芳香醛的克莱森一施密特缩合反应，设计并合成了 系列熊果酸苄基苯胺衍生物, 以期开发出有效的抗肿瘤 分子, 所合成化合物对 4 种人癌细胞系(包括 A-549、 MCF-7、HCT-116、THP-1)和正常人上皮细胞系(FR-2) 的抑制活性评估结果显示, 所有化合物均显示出较高的 抗肿瘤活性, 其中活性最好的化合物 61 对上述癌细胞 的 $\mathrm{IC}_{50}$ 值分别为 $0.55,<0.1,5.5,0.9,0.8 \mu \mathrm{mol} \cdot \mathrm{L}^{-1}$. Finlay 等 ${ }^{[54]}$ 报道了影响 NRP.152 前列腺细胞生长调节的 新型熊果酸 $\mathrm{A}$ 环裂解类似物的合成. 其中化合物 62 对 NRP.152 前列腺细胞增殖抑制作用的 $\mathrm{IC}_{50}$ 值为 2.4 $\mu \mathrm{mol} \cdot \mathrm{L}^{-1}$.

$\mathrm{Gu}$ 等 ${ }^{[55]}$ 设计合成了系列新型 $\mathrm{A}$ 环修饰的熊果酸喹 㕲衍生物, 对三种人癌细胞株(MDA-MB-231、HeLa 和 SMMC-7721)的体外细胞毒性结果显示, 化合物 $63 \mathbf{a} \sim$ 63d 对三种癌细胞株具有明显的抗肿瘤活性, 其中化合
物 63b 对 MDA-MB-231、 HeLa 和 SMMC-7721 细胞的 $\mathrm{IC}_{50}$ 值分别为 $(0.61 \pm 0.07) 、(0.36 \pm 0.05) 、(12.49 \pm 0.08)$ $\mu \mathrm{mol} \cdot \mathrm{L}^{-1}$. 该课题组 ${ }^{[56]}$ 还设计合成了一系列新型咔唑类 熊果酸衍生物, 以期开发出有效的抗菌和抗肿瘤药物, 并对四种细菌和三种真菌菌株进行了抗菌活性评价. 化 合物 64a $\sim 64 b$ 和 65 对至少一种被测细菌具有显著的抗 菌活性，最低抑菌浓度(MIC)值为 $3.9 \sim 15.6 \mu \mathrm{mol} \cdot \mathrm{L}^{-1}$, 上述化合物对两种人类肿瘤细胞系(SMMC-7721 和 HepG2) 显示出明显的细胞毒活性, $\mathrm{IC}_{50}$ 值低于 10 $\mu \mathrm{mol} \cdot \mathrm{L}^{-1}$, 特别是化合物 65a, 对 SMMC-7721 和 HepG2 细胞的 $\mathrm{IC}_{50}$ 值分别为(1.08 \pm 0.22$)$ 和(1.26 \pm 0.17$) \mu \mathrm{mol} \bullet$ $\mathrm{L}^{-1}$, 与阿霉素相当. 此外, 化合物 65a 对非癌 LO2 细胞 的细胞毒性降低, $\mathrm{IC}_{50}$ 值为 $(5.75 \pm 0.48) \mu \mathrm{mol} \cdot \mathrm{L}^{-1}$.

Lin 等 ${ }^{[57]}$ 报道了 UA 查尔酮衍生物 66 在抑制肝干细 胞 $\mathrm{CD} 133^{+}$球形形成细胞特性中的作用，化合物 66 在 $\mathrm{PLC} / \mathrm{PRF} / 5$ 和 Huh7 肝癌细胞中以剂量和时间依赖的方 式抑制 $\mathrm{CD} 133^{+}$的表达. Mendes 等 ${ }^{[58]}$ 报道了系列新型 $\mathrm{A}$ 环裂解 UA 衍生物在人非小细胞肺癌细胞中的合成及细 胞毒活性研究. 其中活性最好的化合物 67 对肺癌细胞 $\mathrm{H} 460, \mathrm{H} 322, \mathrm{H} 460 \mathrm{LKB} 1$ 的 $\mathrm{IC}_{50}$ 值分别为 2.6, 3.3, 4.4 $\mu \mathrm{mol} \cdot \mathrm{L}^{-1}$, 其通过激活 caspase-8、caspase-7 和降低 Bcl-2 诱导细胞调亡. Sun 等 ${ }^{[59]}$ 设计合成了系列熊果酸 A 环修 饰的衍生物, 其中活性最好的化合物 68 对不同癌细胞 抑制活性的 $\mathrm{IC}_{50}$ 值分别为 Hela: $15.1 \mu \mathrm{mol} \cdot \mathrm{L}^{-1}, \mathrm{HepG} 2$ : $16.65 \mu \mathrm{mol} \cdot \mathrm{L}^{-1}$, HT1080: $17.18 \mu \mathrm{mol} \cdot \mathrm{L}^{-1}, \mathrm{MCF}-7: 17.58$ $\mu \mathrm{mol} \cdot \mathrm{L}^{-1}, \mathrm{SK}-\mathrm{N}-\mathrm{MC}: 17.06 \mu \mathrm{mol} \cdot \mathrm{L}^{-1}$, 更重要的是作者 发现了一个新的细胞死亡途径，化合物 68 通过过度刺 激胞饮作用诱导癌细胞死亡. 金晓燕等[60] 以熊果酸为 原料合成了一系列新型的熊果酸喹啉酰肼衍生物 $69 \mathrm{a} \sim$ 69f 和 1,3,4-噁二唑衍生物 $70 a \sim 70 f$. 测试了其对 MDA-MB-231、HeLa 和 SMMC-77213 三种肿瘤细胞株 的增殖抑制活性，其中化合物 69a 69c 对所测试 3 种细 胞株均表现出显著的抑制活性，对 MDA-MB-231 细胞 的 $\mathrm{IC}_{50}$ 值为 $1.16 \sim 1.84 \mu \mathrm{mol} \cdot \mathrm{L}^{-1}$, 对 $\mathrm{HeLa}$ 细胞的 $\mathrm{IC}_{50}$ 值为 $0.83 \sim 1.18 \mu \mathrm{mol} \cdot \mathrm{L}^{-1}$, 对 SMMC-7721 的 $\mathrm{IC}_{50}$ 值为 $17.48 \sim 19.41 \mu \mathrm{mol} \cdot \mathrm{L}^{-1}$, 强于阳性对照依托泊苷. 构效 关系分析发现，在熊果酸分子中引入喹啉基，并将羧基 修饰为酰肼基和 1,3,4-噁二唑基, 能够显著增强其抗肿 瘤活性, 且侧链为酰肼的衍生物活性优于噁二唑的衍生 物.

如前所述, 孟艳秋课题组对熊果酸的结构修饰进行 了大量研究工作, 该课题组 ${ }^{[61]}$ 在熊果酸 A 环骈合 4-氯吲 哚环, 同时对 C-28 位进行成酯、酰胺化等结构修饰, 设 计合成了两类共 10 个新型熊果酸 $\mathrm{A}$ 环衍生物. 所有化 合物对 HepG2、SGC7901 肿瘤细胞的抑制作用均强于 
母体熊果酸, 尤其是化合物 71、72 表现出较为显著的抗 肿瘤活性, 与已上市药物紫杉醇活性相当, 值得进一步 研究. 该课题组的工作还包括在熊果酸 $\mathrm{A}$ 环上引入含 氮、氧杂环, 同时对其 C-28 位羧基进行酰胺化结构修 饰 ${ }^{[62]}$, 选用高表达人口腔表皮样癌细胞 $\mathrm{KB}$ 和人胃癌细 胞 SGC7901 对它们进行初步的体外抗肿瘤活性研究. 上述化合物对 KB 和 SGC7901 肿瘤细胞的抑制活性均 明显强于熊果酸, 其中化合物 73 和 74 对 SGC7901 肿瘤 细胞的抑制效果显著, $\mathrm{IC}_{50}$ 值分别为 0.289 和 $0.04 \mu \mathrm{mol} \cdot$ $\mathrm{L}^{-1}$, 活性明显高于已上市药物吉非替尼 $\left(\mathrm{IC}_{50}=1.03\right.$ $\left.\mu \mathrm{mol} \cdot \mathrm{L}^{-1}\right)$.

王玉英等 ${ }^{[63]}$ 设计、合成了系列喹喔啉熊果酸-查尔 酮缀合物, 其对 MCF-7、PC-3、GBC-823 和 $\mathrm{KB}$ 细胞均 有抑制活性, 尤其是对 MCF-7 细胞的抑制活性与熊果 酸相比, 均有提高, 其中化合物 $75 \mathrm{a}\left(\mathrm{IC}_{50}=14.2 \mu \mathrm{mol}\right.$ • $\left.\mathrm{L}^{-1}\right)$, 75b $\left(\mathrm{IC}_{50}=15.3 \mu \mathrm{mol} \cdot \mathrm{L}^{-1}\right)$ 和 $75 \mathbf{c}\left(\mathrm{IC}_{50}=10.6\right.$ $\mu \mathrm{mol} \cdot \mathrm{L}^{-1}$ ) 对 MCF-7 细胞的抑制活性甚至强于临床上应 用药物他莫昔芬 $\left(\mathrm{IC}_{50}=15.9 \mu \mathrm{mol} \cdot \mathrm{L}^{-1}\right)$, 并且这些缀合 物对正常的乳腺上皮细胞 MCF-10A 没有毒性. 该课题 组 ${ }^{[64]}$ 还合成了系列含有杂环的熊果酸衍生物, 其对所 测试的五种肿瘤细胞均有抑制活性, 尤其是喹喔啉熊果 酸 75b 对 MCF-7、GBC-823 和 KB 肿瘤细胞的抑制活性 均强于先导化合物熊果酸, 且这些杂环熊果酸对正常乳
腺上皮细胞 $\mathrm{MCF}-10 \mathrm{~A}$ 没有毒性.

\section{2 熊果酸的结构修饰与抗糖尿病和抗心血管疾 病活性}

熊果酸衍生物在抗糖尿病方面的研究, 以蛋白酪氨 酸磷酸酶 $1 \mathrm{~B}$ 和 $\alpha$-葡萄糖苷酶为靶点合成了部分有抗糖 尿病潜力的 UA 衍生物. 另外, UA 衍生物在心血管疾病 的预防和治疗方面也有报道.

蛋白酪氨酸磷酸酶 1B (PTP-1B)作为治疗 2 型糖尿 病的一个新靶点引起了人们的兴趣, 这是因为它在胰岛 素信号通路中作为一种负调节因子的作用. 因此, 研究 者设计合成了 PTP-1B 的抑制剂熊果酸衍生物 76 作为潜 在的抗糖尿病药物 ${ }^{[65]}$, 该化合物对 PTP-1B 的抑制浓度 $\mathrm{IC}_{50}=283 \mathrm{nmol} \cdot \mathrm{L}^{-1}$. Guzmán-Ávila 等[66]也报道了 UA 衍 生物对 PTP-1B 的抑制作用，其中化合物 77a、77b 和 78 的 $\mathrm{IC}_{50}$ 值分别为 5.6、4.7 和 $4.6 \mu \mathrm{mol} \cdot \mathrm{L}^{-1}$. 化合物 $77 \mathrm{c}$ 和 78 的体内抗糖尿病作用显示, 采用非胰岛素依赖性 糖尿病小鼠模型，与对照组相比，药物组能显著降低非 胰岛素依赖性糖尿病小鼠的血糖浓度. Kazmi 等 ${ }^{[67]}$ 报道, 熊果酸的硬脂酰葡萄糖苷 79 在链脲佐菌素诱导的糖尿 病大鼠中也表现出显著的降血糖活性，具有潜在的抗糖 尿病的潜力(图 6).

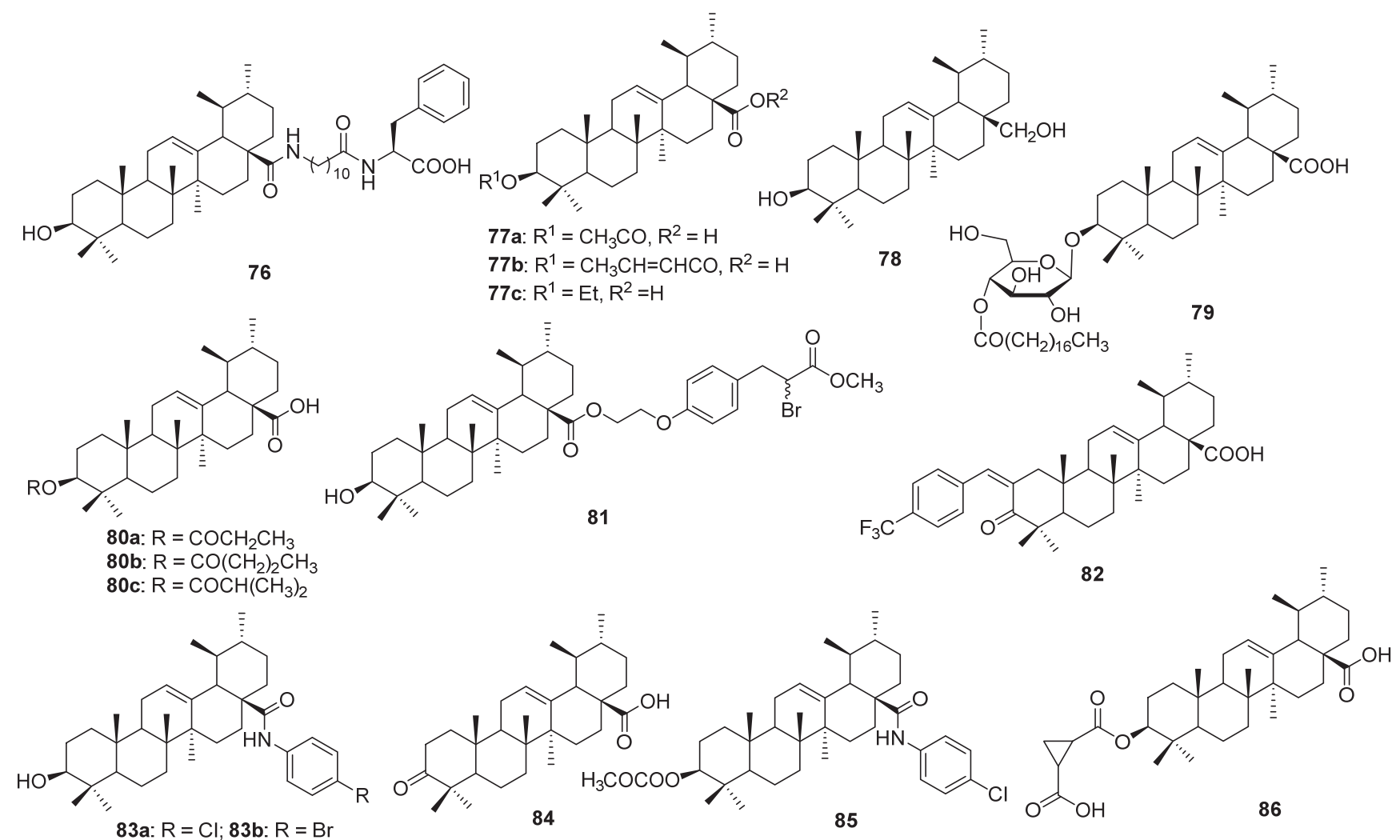

图 6 具有抗糖尿病和心血管疾病活性的熊果酸衍生物结构

Figure 6 Structures of ursolic acid derivatives with anti-diabetes activity and anti-cardiovascular activity 
$\mathrm{Wu}$ 课题组 ${ }^{[68-70]}$ 对熊果酸衍生物的抗糖尿病活性进 行了大量研究, 先后合成了系列具有抗糖尿病活性的新 型 UA 衍生物, 并对其体外 $\alpha$-葡萄糖苷酶抑制活性进行 了篮选, 大多数 UA 衍生物均表现出明显的抑制活性, 尤其是化合物 80a $\sim 80 \mathrm{c}$ 和 81 , 对大鼠心肌细胞有显著 的抑制作用, 其 $\mathrm{IC}_{50}$ 值分别为 $2.66 、 1.01 、 3.26$ 、和 3.24 $\mu \mathrm{mol} \cdot \mathrm{L}^{-1}$, 这些化合物对 $\alpha$-葡萄糖苷酶的抑制活性均强 于阳性对照阿卡波糖[68]. 另外熊果酸衍生物 82 对 $\alpha$-葡 萄糖苷酶抑制活性的 $\mathrm{IC}_{50}$ 值为 $(0.71 \pm 0.27) \mu \mathrm{mol} \cdot \mathrm{L}^{-1}$, 比其他类似物和阳性对照物有更好的抑制活性 ${ }^{[69]}$. 该 课题组还设计合成了 UA 衍生物 83a 和 $83 \mathrm{~b}$, 具有良好 的 $\alpha$-葡萄糖苷酶抑制活性, $\mathrm{IC}_{50}$ 值分别为 $(1.27 \pm 0.27)$ 和 $(1.28 \pm 0.27) \mu \mathrm{mol} \cdot \mathrm{L}^{-1}$, 均低于其他类似物和阳性对照 组 ${ }^{[70]}$. 陈否等 ${ }^{[71]}$ 合成的熊果酸衍生物 84 同样具有较好 的 $\alpha$-葡萄糖苷酶的抑制活性, $\mathrm{IC}_{50}$ 值为 $8.7 \mu \mathrm{mol} \cdot \mathrm{L}^{-1}$. Zhang 等 ${ }^{[72]}$ 报道了一系列取代的五环三萜类化合物的 合成及其作为潜在的 PPAR $\gamma$ 激动剂和糖原磷酸化酶抑 制剂的活性, 其中三萜熊果酸衍生物 85 的抑制作用最 强, 对家兔肌肉糖原磷酸化酶 $\mathrm{A}(\mathrm{RMGPa})$ 的 $\mathrm{IC}_{50}$ 值为 $40.6 \mu \mathrm{mol} \cdot \mathrm{L}^{-1}$.

在心血管疾病的预防和治疗中, 胆固醇输送蛋白 (CETP)通过降低低密度脂蛋白胆固醇水平和提高人体 血浆中高密度脂蛋白胆固醇水平, 成为一种有吸引力的 治疗靶点. Chen 等 ${ }^{[73]}$ 合成了系列熊果酸 $3 \beta$ 酯衍生物, 并考察了其对 CETP 的抑制活性. 其中衍生物 86 的作用 最强, 对 CETP 抑制作用 $\mathrm{IC}_{50}$ 值为 $2.4 \mu \mathrm{mol} \cdot \mathrm{L}^{-1}$.

\section{3 熊果酸的结构修饰与抗炎活性}

以提高抗炎为目的对熊果酸的结构修饰的研究也 很多, 主要集中在 C-2 位、C-28 位、C 环, A 环的修饰. 对这些位点的修饰取得了重大的进步, 获得了数个活性 优良的化合物(图 7), 部分化合物具有进一步开发应用 的前景.

Wei 等 ${ }^{[74]}$ 报道了含氮杂环熊果酸衍生物作为抗炎抑 制剂的设计、合成及活性评价，合成了含有噁二唑、三 唑酮和哌嗪的熊果酸衍生物, 其中化合物 $87 \mathrm{~b}$ 的抗炎活 性最强, 腹腔给药后抑制率为 $69.76 \%$, 明显高于对照药 消炎痛和布洛芬. 噻唑蓝(MTT)法测定了化合物的细胞 毒性, 无明显的细胞毒性 $\left(\mathrm{IC}_{50}>100 \mu \mathrm{mol} \cdot \mathrm{L}^{-1}\right)$. Nelson 等 ${ }^{[75]}$ 报道了具有抗炎作用的熊果酸含氧衍生物的合成, 对其在化学诱导的皮肤炎症小鼠模型中抑制 TPA 诱导 的表皮炎症细胞因子 mRNA 表达的能力评估结果显示, 化合物 88 和 89 抑制 IL-1 $\alpha$ 、IL-1 $\beta$ 、IL-6 和 IL-23 的表 达效果最强, 且都好于熊果酸本身. Zhang 等[76]研究了 熊果酸在巨型芽孢杆菌 CGMCC 1.1741 上的微生物转
化, 得到 5 种代谢产物 $90 \sim 94$. 在脂多糖(LPS)刺激的 RAW 264.7 炎症模型中, 5 种代谢产物均表现出不同程 度的抑制一氧化氮(NO)生成活性，其中化合物 92 和 94 的 $\mathrm{IC}_{50}$ 值分别为 1.24 和 $1.71 \mu \mathrm{mol} \cdot \mathrm{L}^{-1}$, 是很有前途的治 疗 NO 过量引起的炎症性疾病的候选化合物. 李财虎

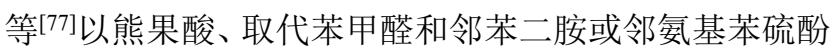
为原料, 合成了一系列熊果酸苯并咪(噻)唑连苯酯衍生 物 95a 95d 和 96a 96d, 其中化合物 95d (150 mg・ $\left.\mathrm{kg}^{-1}\right), 96 \mathrm{c}\left(150 \mathrm{mg} \cdot \mathrm{kg}^{-1}\right)$ 具有良好的抗炎活性. 谭娟 等 ${ }^{[78]}$ 以熊果酸、对羟基苯乙酮和芳醛为原料合成了一系 列熊果酸衍生物一查尔酮缀合物 $97 \mathrm{a} \sim 97 \mathrm{~g}, 98 \mathrm{a} \sim 98 \mathrm{~g}$, 99a 99g, 上述化合物对二甲苯致小鼠耳廓肿胀和角叉 菜胶大鼠足跖肿胀炎症模型皆有不同程度的抗炎活性, 部分化合物的抗炎活性与熊果酸相比均有提高. $\mathrm{Li}$ 等 ${ }^{[79]}$ 报道了系列 C-3 位取代的熊果酸衍生物对 PM2.5 诱导的 慢性阻塞性肺病(COPD)的预防作用，包括抑制肺部炎 症作用, 其中化合物 100 在治疗 PM2.5 诱导的 COPD 小 鼠肺部炎症作用中，炎症因子 TNF- $\alpha$ 和 IL-6 等的水平降 低最为显著.

\section{4 熊果酸的结构修饰与预防骨损伤活性}

色氨酸差化酶 1 (TPH-1)是外周血清素生物合成的 主要酶, 由于外周血清素抑制骨形成, TPH-1 抑制剂提 供了一个有用的工具来发现骨质疏松症，为骨质疏松症 的合成代谢药物设计提供了新的靶点. 作者 ${ }^{[80-81]}$ 以分子 对接技术为指导，设计合成了一系列新的熊果酸衍生 物, 其中 101 和 102 作为有效的 TPH-1 结合剂, 对 5-差弪 色胺的合成有较强的抑制作用，能抑制 TPH-1 蛋白和 mRNA 的表达, 降低血清和肠道中 5-差色胺的含量, 而 对脑 5-差色色胺无影响, 显著预防骨丢失, 且无雌激素副 作用.

$\mathrm{Yu}$ 等 ${ }^{[82]}$ 报道了熊果酸衍生物 103 可通过改善糖尿 病小鼠脂肪细胞功能障碍，促进新骨形成及抑制破骨细 胞的吸收功能, 从而改善链脲佐菌素所致的骨损伤.

\section{5 熊果酸的结构修饰与抗病原微生物活性}

熊果酸衍生物具有优异的抗菌、抗病毒等抗微生物 活性, 研究者对熊果酸及其衍生物在抗病原微生物方面 进行了大量研究, 获得了许多有进一步开发前景的衍生 物(图 9).

Kong 课题组 ${ }^{[83]}$ 研究结果首次表明, 天然产物熊果 酸可以作为潜在的丙型肝炎病毒 $(\mathrm{HCV})$ 抗病毒药物应用 于临床试验, 无论是作为单一疗法还是与其他 $\mathrm{HCV}$ 抗 病毒药物联合应用. Zhong 等 ${ }^{[84]}$ 报道了以拉米夫定和熊 果酸为原料, 通过酰胺和酯键与氯乙酸乙酯偶联, 合成 


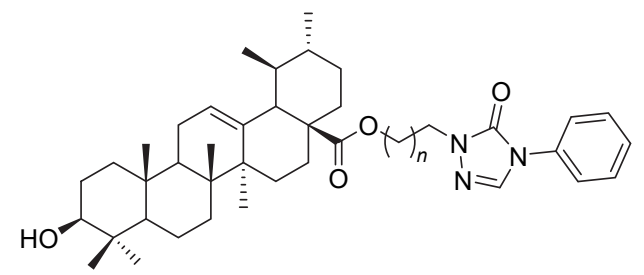

87a $\sim 87 \mathrm{~d}: n=1,2,3,4$

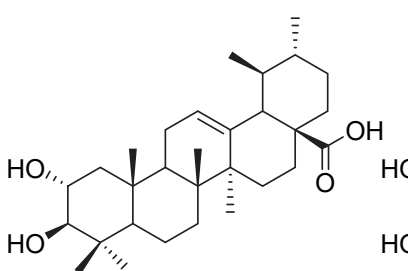

88

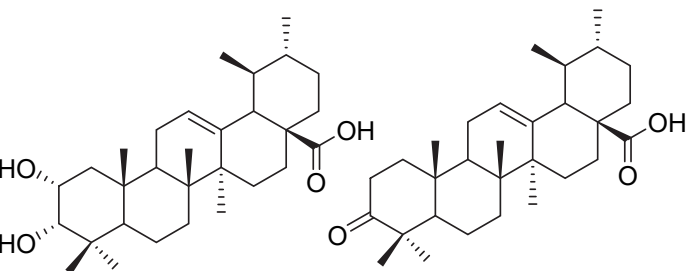

89
90

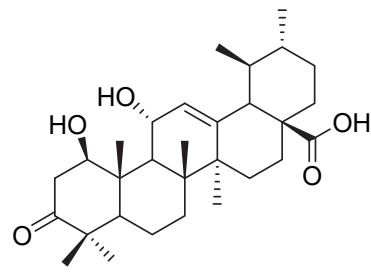

91

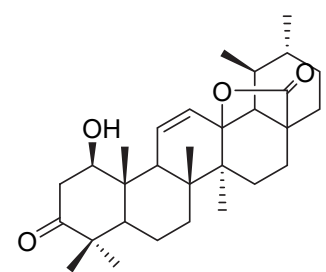

92

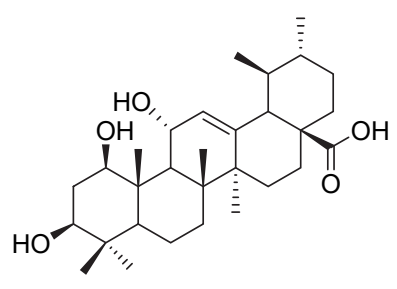

93

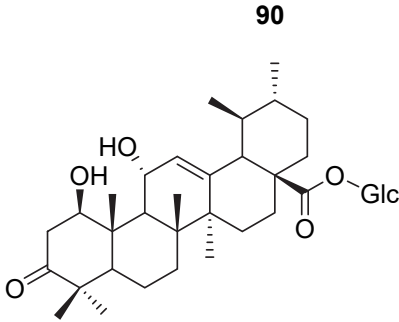

94

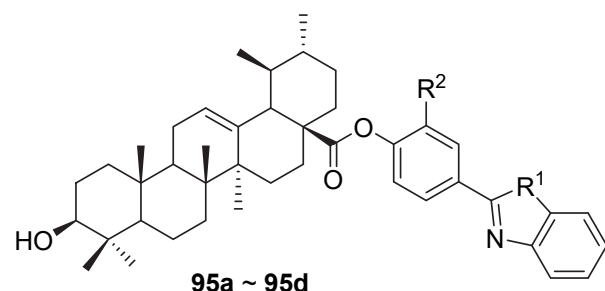
a: $R^{1}=N H, R^{2}=H ; b: R^{1}=S, R^{2}=H$; c: $\mathrm{R}^{1}=\mathrm{NH}, \mathrm{R}^{2}=\mathrm{OCH}_{3} ; \mathbf{d}: \mathrm{R}^{1}=\mathrm{S}, \mathrm{R}^{2}=\mathrm{OCH}_{3}$

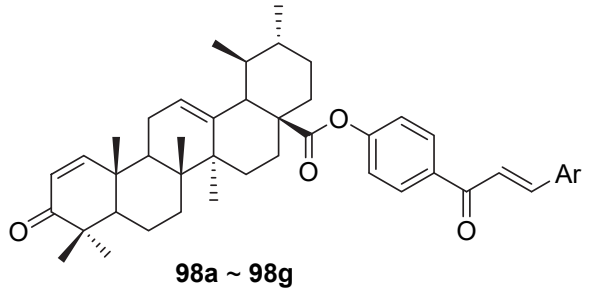

a $\sim$ g: $\mathrm{Ar}=\mathrm{Ph}, 3-\mathrm{MeOC}_{6} \mathrm{H}_{4} .4-\mathrm{MeOC}_{6} \mathrm{H}_{4}$, 4- $\mathrm{FC}_{6} \mathrm{H}_{4}, 4-\mathrm{ClC}_{6} \mathrm{H}_{4}$, 2-fury, 2-thienyl

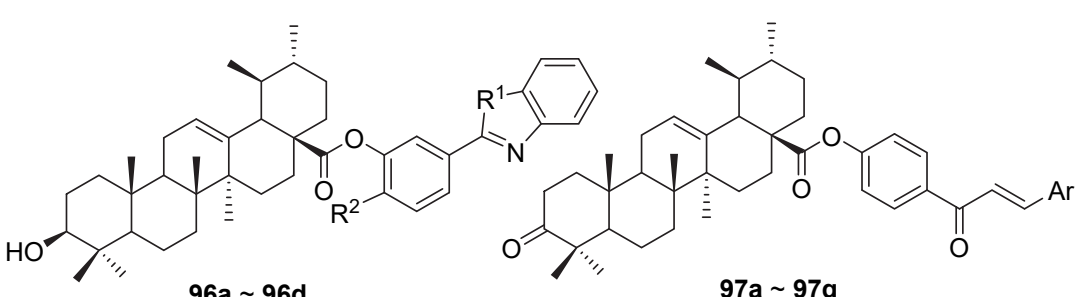

$96 a \sim 96 d$

a: $\mathrm{R}^{1}=\mathrm{NH}, \mathrm{R}^{2}=\mathrm{H} ; \mathbf{b}: \mathrm{R}^{1}=\mathrm{S}, \mathrm{R}^{2}=\mathrm{H}$; c: $\mathrm{R}^{1}=\mathrm{NH}, \mathrm{R}^{2}=\mathrm{OCH}_{3} ; \mathbf{d}: \mathrm{R}^{1}=\mathrm{S}, \mathrm{R}^{2}=\mathrm{OCH}_{3}$

$\mathbf{a} \sim \mathbf{g}: \mathrm{Ar}=\mathrm{Ph}, 3-\mathrm{MeOC}_{6} \mathrm{H}_{4} .4-\mathrm{MeOC}_{6} \mathrm{H}_{4}$

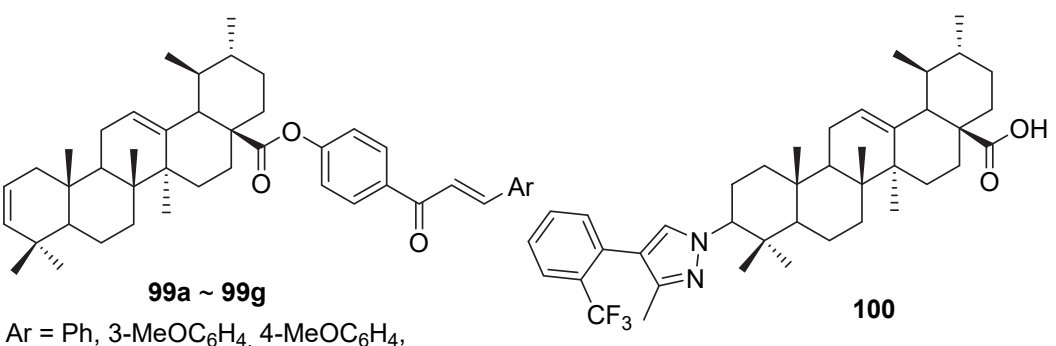

$\mathrm{Ar}=\mathrm{Ph}, 3-\mathrm{MeOC}_{6} \mathrm{H}_{4}$ 4- $\mathrm{MeOC}_{6} \mathrm{H}_{4}$, 4- $\mathrm{FC}_{6} \mathrm{H}_{4}, 4-\mathrm{ClC}_{6} \mathrm{H}_{4}$, 2-fury, 2-thienyl

图 7 具有抗炎活性的熊果酸衍生物的结构

Figure 7 Structures of ursolic acid derivatives with anti-inflammatory activity

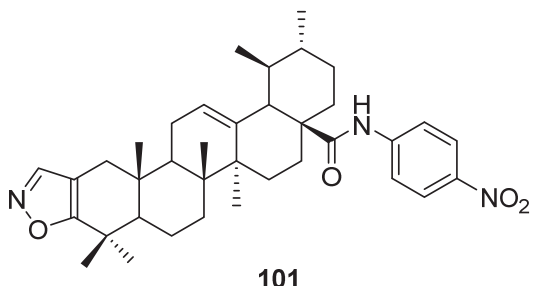

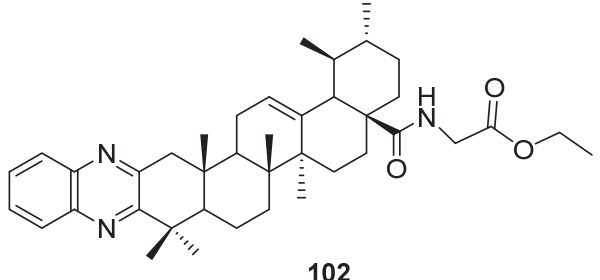<smiles>CCC(=O)OC1CCC2(C)C(CC=C3C4C(C)CC[C@@H](C)C4(C(=O)O)CCC32C)C1(C)C</smiles>

图 8 具有预防骨损伤活性的熊果酸衍生物的结构

Figure 8 Structure of ursolic acid derivatives with bone protective activity

了一种新型孪药 104, 衍生物 104 具有抗乙型肝炎病毒 活性和抗急性肝损伤的双重作用, 是一个有前途的治疗 肝炎的候选药物.

$\mathrm{Li}$ 等 ${ }^{[85}$ 合成了系列新型 $\mathrm{H} 5 \mathrm{~N} 1$ 病毒入侵抑制剂熊果 酸衍生物, 其中化合物 $\mathbf{1 0 5}$ 表现出最强的抗 H5N1 效力, 无细胞毒性, 超过利巴韦林的效力. Song 等[86]基于先前 发现的一种小分子 $\mathrm{H} 5 \mathrm{~N} 1$ 抑制剂皇式 106, 设计、合成
了一系列熊果酸甲酯 3- $O-\beta$-木瓜苷类似物, 所有化合物 均表现出对 $\mathrm{H} 5 \mathrm{~N} 1$ 病毒的抑制活性，其效价范围从中等 $\left(\mathrm{IC}_{50}>10 \mu \mathrm{mol} \cdot \mathrm{L}^{-1}\right)$ 到强 $\left(\mathrm{IC}_{50}<4 \mu \mathrm{mol} \cdot \mathrm{L}^{-1}\right)$, 与阳性对 照药 $\mathrm{IC}_{50}$ 值相当. 3- $O-\beta$-木瓜苷熊果酸衍生物作为新型 $\mathrm{H} 5 \mathrm{~N} 1$ 抑制剂的构效关系研究结果表明, 熊果酸 C-28 位 羧基处的酰胺结构或熊果酸 C-3 构型由 $3 \beta$ 型改为 $3 \alpha$ 型, 有助于显著提高选择性指数, 同时保持其抗病毒活性. 


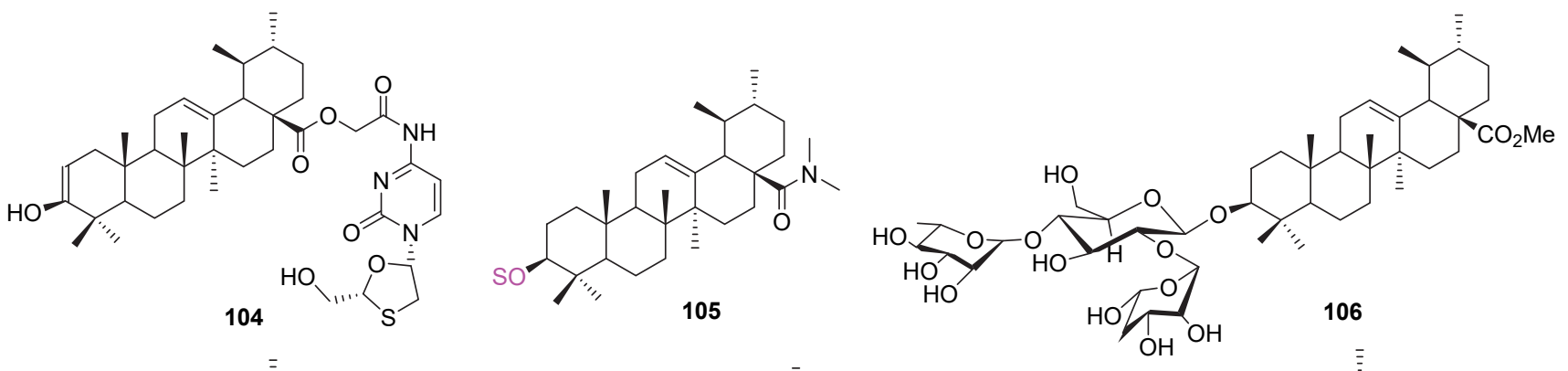

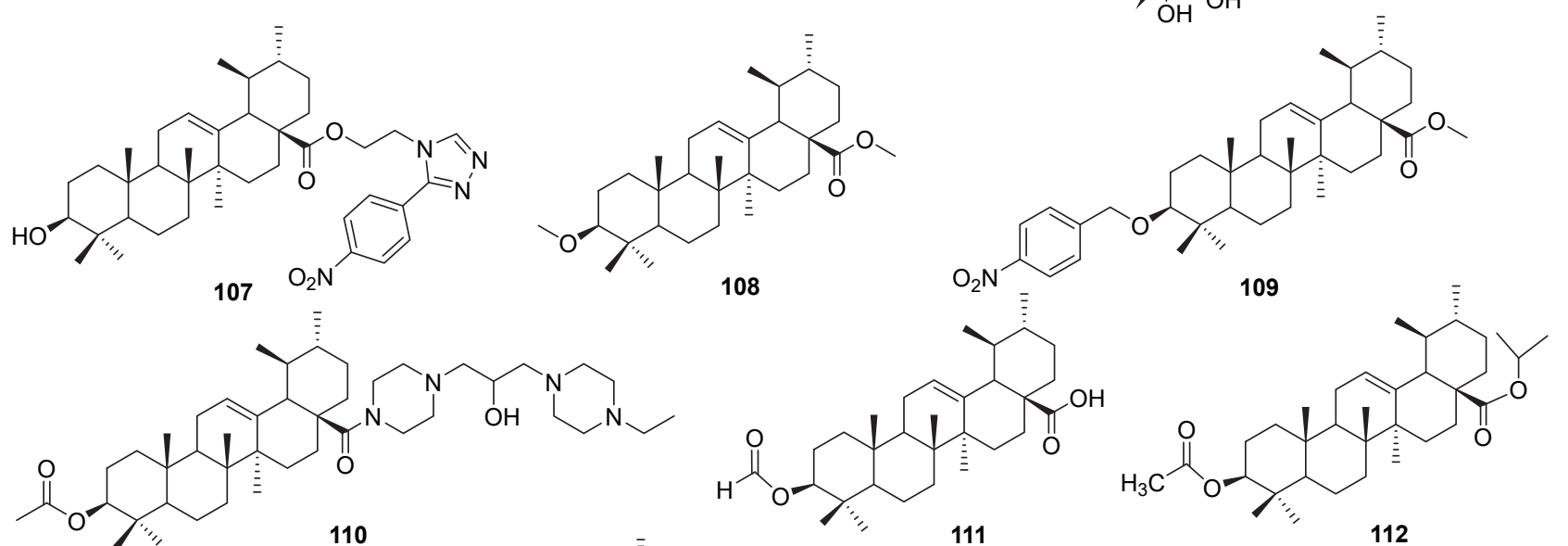

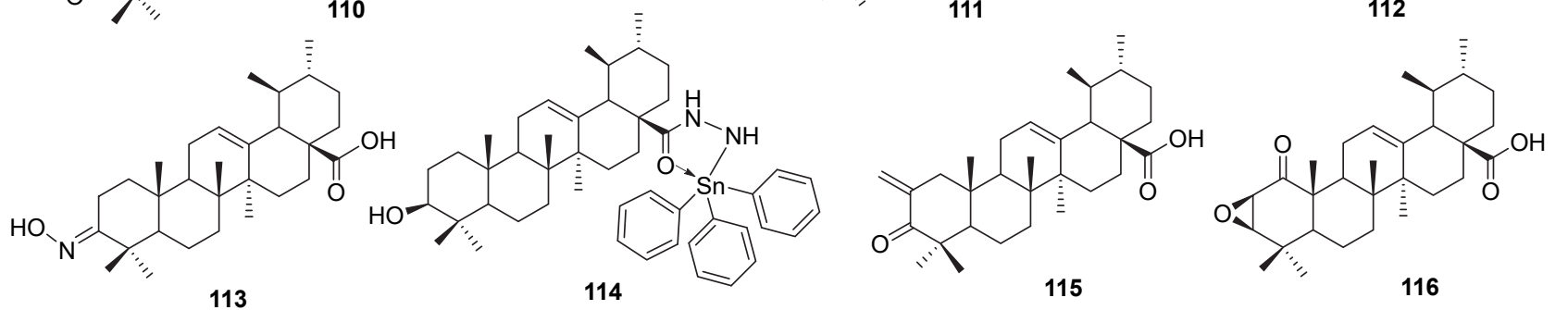

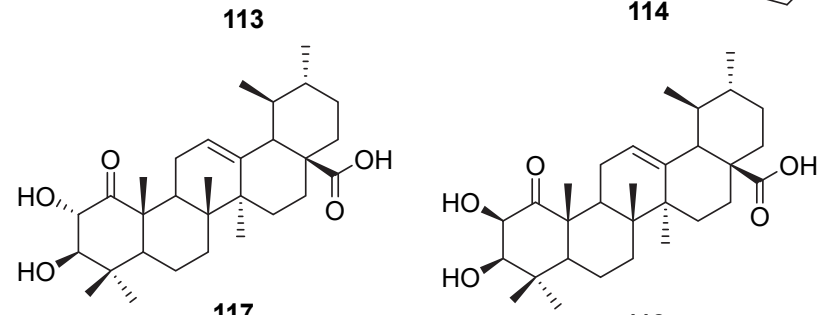

117

118

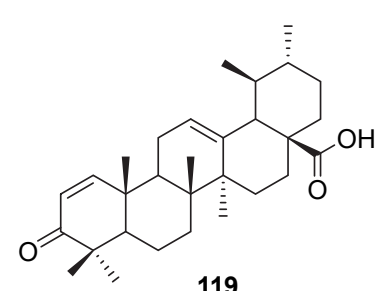

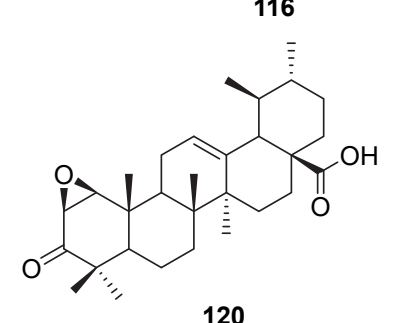

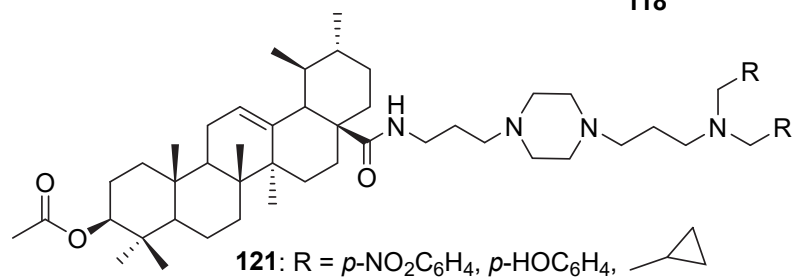

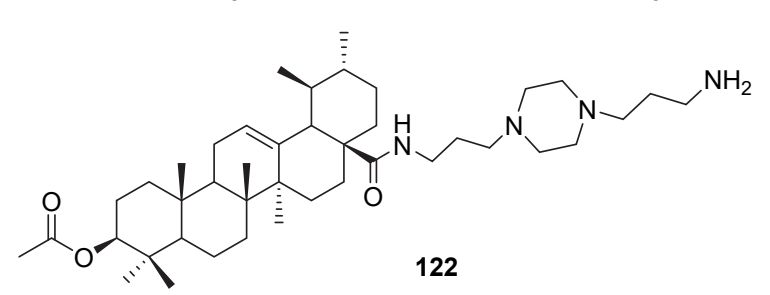

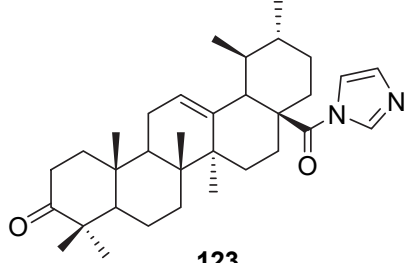

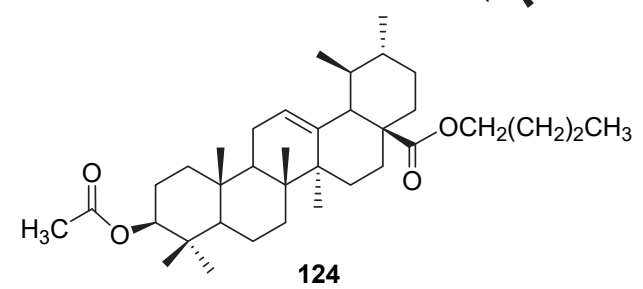

图 9 具有抗病原微生物活性的熊果酸衍生物的结构

Figure 9 Structures of ursolic acid derivatives with antimicrobial activity

Luan 等 ${ }^{[87]}$ 合成了三种新型含三唑环的熊果酸类抗 抗弓形虫活性和较低的细胞毒性, 其中化合物 107 体内 弓形虫衍生物, 所合成 UA 衍生物大多数表现出较好的抗弓形虫活性最强. 
目前临床治疗棘阿米巴角膜炎的药物少, 疗效差, 函需新型的抗棘阿米巴药物. 熊果酸具有广泛的抗棘阿 米巴活性. Sifaoui 等 ${ }^{[8]}$ 合成了系列熊果酸衍生物, 其水 溶性较熊果酸有明显改善, 同时所有衍生物均显示出比 UA 更强的抗棘阿米巴活性, 其中化合物 108 和 109 对 刺阿米巴具有较强的抗菌活性, 活性高且毒性较低.

Wang 等 ${ }^{[89}$ 合成了系列具有显著体外抗菌活性的新 型哌嗪类熊果酸杂化物. 新型哌嗪类熊果酸杂化物能显 著抑制水稻白叶枯病菌和柑桔黄叶枯病菌的生长, 活性 最好的化合物 110 对上述两种菌的 $\mathrm{EC}_{50}$ 值分别为 0.37 和 $1.08 \mu \mathrm{mol} \cdot \mathrm{L}^{-1}$, 显著超过 UA $\left(>400 \mu \mathrm{mol} \cdot \mathrm{L}^{-1}\right)$ 和阳 性对照. Cunha 等 ${ }^{[00]}$ 报道了系列三萜酸及其半合成衍生 物对口腔致病菌的抗菌活性, 所评价的微生物包括: 变 形链球菌、米特链球菌, 血链球菌, 唾液链球菌、远缘 链球菌和粪肠球菌, 它们可能导致人类龉齿的形成. 其 中熊果酸和齐墩果酸具有最强的抗菌作用, MIC 值在 $30 \sim 80 \mu \mathrm{mol} \cdot \mathrm{L}^{-1}$ 之间. 熊果酸衍生物以及熊果酸和齐 墩果酸混合物表现出的抗菌活性不如单独用熊果酸或 齐墩果酸的抗菌活性强, 构效关系研究发现三萜中的羟 基和羧基对其抗菌活性有重要影响. 2014 年 Do Nascimento 等 ${ }^{[91]}$ 从桑树地上部分乙醇提取物中分离得 到一种重要的生物活性物质熊果酸, 通过 C-3 改性得到 了两个半合成化合物. 研究了熊果酸及其衍生物的抑菌 和抗氧化活性, 其中化合物 111 与卡那霉素联合治疗痰 中多药耐药临床分离株大肠杆菌, MIC 值由 $128 \mu \mathrm{g} / \mathrm{mL}$ 降至 $8 \mu \mathrm{g} / \mathrm{mL}$, 协同作用显著. 1,1-二苯基-2-三硝基苯肼 (DPPH)法测定结果显示其具有显著清除自由基的活性.

Dwivedi 等 ${ }^{[92]}$ 报道了熊果酸衍生物对多药耐药大肠 杆菌的耐药性逆转的研究, 评估了熊果酸的六种半合成 衍生物结合常规抗生素荎啶酸(NA) 对菜啶酸敏感 (NASEC) 和䒺啶酸耐酸(NAREC) 菌株大肠杆菌的抗菌 和耐药逆转潜力. 虽然 UA 及其所有半合成衍生物均未 显示出自身的抗菌活性, 但它们的结合使 NA 的 MIC 显 著降低了 8 倍, 其中 UA 衍生物 112 和 113 分别使 NA 对 NASEC 的 MIC 降低 8 倍和对 NAREC 的 MIC 降低 4 倍和 8 倍, 进一步评价了 112 和 113 与另一种抗生素四 环素(TET)对大肠杆菌(MDREC-KG4)多药耐药临床分 离株的协同作用, 这两种衍生物与 TET 联用均能显著 抑制外排泵, 使细胞活力呈浓度依赖性下降. 这些 UA 衍生物可能在治疗多药耐药革兰阴性感染中有潜在的 协同作用.

Jabeen 等 ${ }^{[93]}$ 以熊果酸的酰肼衍生物为原料, 合成了 与锡、锑、铁的稀土金属配合物, 并对其进行了抗菌和 抗氧化活性研究. 这些金属配合物比母体化合物具有更 强的抗氧化能力, 对两株革兰氏阴性和两株革兰氏阳性
细菌评价结果表明, 三苯基锡络合物 114 对志贺氏菌、 伤寒沙门菌和金黄色葡萄球菌的 MIC 值均为 $8 \mu \mathrm{g} / \mathrm{mL}$. 同时, 对肺炎链球菌的 MIC 值为 $4 \mu \mathrm{g} / \mathrm{mL}$. 如前所述, $\mathrm{Gu}$ 等 ${ }^{[56]}$ 设计合成了一系列新型咔唑类熊果酸衍生物, 以期开发出有效的抗菌和抗肿瘤药物, 并对四种细菌和 三种真菌菌株进行了抗菌活性评价, 其中 $63 \mathrm{a}, 63 \mathrm{~b}$ 和 64 系列化合物对至少一种被测细菌具有显著的抗菌活性, $\mathrm{MIC}$ 值为 $3.9 \sim 15.6 \mu \mathrm{mol} \cdot \mathrm{L}^{-1}$. Huang 等 ${ }^{[94]}$ 报道了以熊果 酸、齐墩果酸、甘草次酸、白桦酸为代表，引入 2-亚甲 基-3-氧基作为外环 $\alpha, \beta$-不饱和酮基, 对其进行改性, 并 与母体化合物进行抗菌和抗肿瘤活性比较, 在环 $\alpha$ 位含 有 2-亚甲基-3-氧基的五环三萜类化合物对革兰氏阳性 菌、金黄色葡萄球菌和枯草芽狍杆菌的抗菌活性有显著 提高, 体外对白血病、肺癌和乳腺癌细胞株的剂量依赖 性也表现出增加的细胞毒性, 其中熊果酸衍生物 115 对 肺癌细胞有较强的调亡活性. 孟英才等 ${ }^{[95]}$ 以熊果酸为 原料合成了 $\mathrm{A}$ 环修饰的熊果酸衍生物, 大部分衍生物对 大肠杆菌、铜绿假单胞菌、金黄色葡萄球菌、表皮葡萄 球菌、藤黄微球菌和枯草杆菌这 6 种细菌显示出很强的 抗菌活性, 其中化合物 116 对金黄色葡萄球菌的 MIC 值 为 $6.3 \mu \mathrm{mol} \cdot \mathrm{L}^{-1}$, 化合物 117 和 118 对藤黄微球菌的 MIC 值分别为 12.5 和 $6.3 \mu \mathrm{mol} \cdot \mathrm{L}^{-1}$, 化合物 119 对六个菌种 的 MIC 值均为 $3.1 \mu \mathrm{mol} \cdot \mathrm{L}^{-1}$, 化合物 $\mathbf{1 2 0}$ 对大肠杆菌、 铜绿假单胞菌、金黄色葡萄球菌、表皮葡萄球菌的 MIC 值为 $3.1 \mu \mathrm{mol} \cdot \mathrm{L}^{-1}$. 证明熊果酸 $\mathrm{A}$ 环结构修饰能够有效 提高其抗菌活性, 尤其是 $\mathrm{A}$ 环引入双键或环氧基, 可为 熊果酸抗菌衍生物的进一步研发提供依据.

超过 $40 \%$ 的世界人口有感染疮疾的危险, 疮疾主要 影响热带和亚热带地区的贫困人口. 临床抗疮药物治疗 已利用了植物衍生产品, 如奎宁和青蒿素及其衍生物. 然而, 在全世界范围内使用这些抗疮药已导致抗药性症 原虫的传播, 导致症疾发病率和死亡率增加. Gnoatto 课 题组 ${ }^{[96-100]}$ 对熊果酸的抗寄生虫及症原虫活性进行了大 量研究, 2008 年该课题组 ${ }^{[96]}$ 利用从南美冬青中提取的熊 果酸和齐墩果酸, 合成了一系列新的熊果酸和齐墩果酸 衍生物. 这些新化合物对亚马逊利什曼原虫和贞儿利什 曼原虫株的体外抗寄生虫活性测试结果表明, 一些化合 物对黄曲霉原鞭毛体有抑制作用, IC 50 范围为 $5 \sim 12$ $\mu \mathrm{mol} \cdot \mathrm{L}^{-1}$. 正如作者所料, 大多数化合物对单核细胞具 有显著的细胞毒性 $\left(\mathrm{IC}_{50}=2 \sim 50 \mu \mathrm{mol} \cdot \mathrm{L}^{-1}\right)$. 从构效关系 的角度, 上述药理学结果主要启发了作者对双(3-氨基 丙基)哌嗪熊果酸衍生物 121 及其抗亚马逊河菌株活性 的研究. 2012 年该课题组 ${ }^{[97]}$ 以氯喹敏感的恶性疮原虫 $3 \mathrm{D} 7$ 菌株为研究对象, 在熊果酸 C-3 和 C-28 修饰得到的 类似物中, 化合物 122 表现出卓越的抗 3D7 菌株活性, 
$\mathrm{IC}_{50}$ 为 $175 \mathrm{nmol} \cdot \mathrm{L}^{-1}$, 其通过钙调节途径发挥抗㾏活性. 2013 年该课题组 ${ }^{[98]}$ 在上述基础上又合成了新系列的 C-3 酯基 UA 衍生物, 以期提高其抗㾏疾活性, 降低疮 疾的发病率. 在合成的系列衍生物中, 短侧链对恶性㾏 原虫 3D7 最有效, 无细胞毒性. 这些衍生物的抗症活性 是 UA 的 3 至 5 倍, 并证明线粒体不参与其作用机制, 活 性衍生物对 $\beta$-血红素形成的抑制作用弱于氯喹. 该课题 组 ${ }^{[99]}$ 又于 2017 年报道了系列熊果酸经 C-3 和 C-28 位修 饰后的衍生物, 并证明了它们对抗氯喹恶性疮原虫(W2 株)的抗㾏活性, 部分熊果酸衍生物表现出较好的抗疮 活性, 其中衍生物 123 对恶性㾏原虫 $\mathrm{W} 2$ 的 $\mathrm{IC}_{50}$ 值为 $9.07 \mu \mathrm{mol} \cdot \mathrm{L}^{-1}$. 该课题组 ${ }^{[100]}$ 还报道了对熊果酸衍生物 抗阴道毛滴虫活性的研究. 阴道毛滴虫是一种细胞外寄 生虫, 与人类泌尿生殖道上皮结合，引起性传播感染， 即滴虫病. 鉴于对属于 5-硝基咪唑类药物的耐药性增 加, 迫切需要新的治疗替代品. 作者对 8 个半合成的三 萜衍生物进行了体外抗阴道毛滴虫活性评价. 其中熊果 酸衍生物 124 与其他衍生物相比, 具有最佳的抗阴道 $\mathrm{T}$ 细胞活性, 最小抑菌浓度 $\mathrm{MIC}$ 为 $25 \mu \mathrm{mol} \cdot \mathrm{L}^{-1}$, 化合物 124 对几个阴道新菌临床分离株都有活性. 溶血试验显 示其溶血作用较低. 重要的是, $25 \mu \mathrm{mol} \cdot \mathrm{L}^{-1}$ 化合物 124 对韦罗细胞系没有细胞毒性. 作者还证明了化合物 124 与甲硝唑对一株阴道念珠菌甲硝唑耐药株有协同作用. 以上研究揭示了熊果酸衍生物 124 作为杀滴虫剂的高潜 力.

\section{6 熊果酸的结构修饰与抗神经系统疾病活性}

三萜类化合物作为胆碱酯酶抑制剂的作用受到了 广泛的关注. 这些抑制剂作为改善老年痴呆症, 特别是 阿尔茨海默病患者生活质量的药物仍然很有前景. 有少 量 UA 衍生物被报道具有良好的抗神经系统疾病活性.

Loesche 等[101]报道了具有胆碱酯酶抑制潜力的熊 果酸衍生物的合成，对所合成衍生物进行了抑制酰基胆 碱酯酶和/或丁基胆碱酯酶能力的测定, 熊果酸衍生的 着丙基衍生物 125 是丁酰胆碱酯酶的竞争性抑制剂, 抑 制常数 $K_{\mathrm{i}}=4.29 \mu \mathrm{mol} \cdot \mathrm{L}^{-1}$, 其活性是金标准氢溴酸加兰 他敏的两倍. 熊果酸衍生物 126 具有最佳的乙酰胆碱酯 酶抑制活性，作为一种混合型抑制剂，其对上述两种酶 的抑制常数分别为 $K_{i}=1.72 \mu \mathrm{mol} \cdot \mathrm{L}^{-1}, K_{i}^{\prime}=1.28 \mu \mathrm{mol} \bullet$ $\mathrm{L}^{-1}$.

Kazmi 课题组 ${ }^{[102-104]}$ 报道了熊果酸硬脂酰葡萄糖苷 的抗脑部疾病活性. 揭示了从马缨丹中提取的熊果酸硬 脂酰葡萄糖苷 127 通过促进 GABA 的传递而具有潜在 的抗癫㾁活性, 还有抗惊厥和抗抑郁样活性及抗焦虑活 性，其作用机制还有待进一步研究.

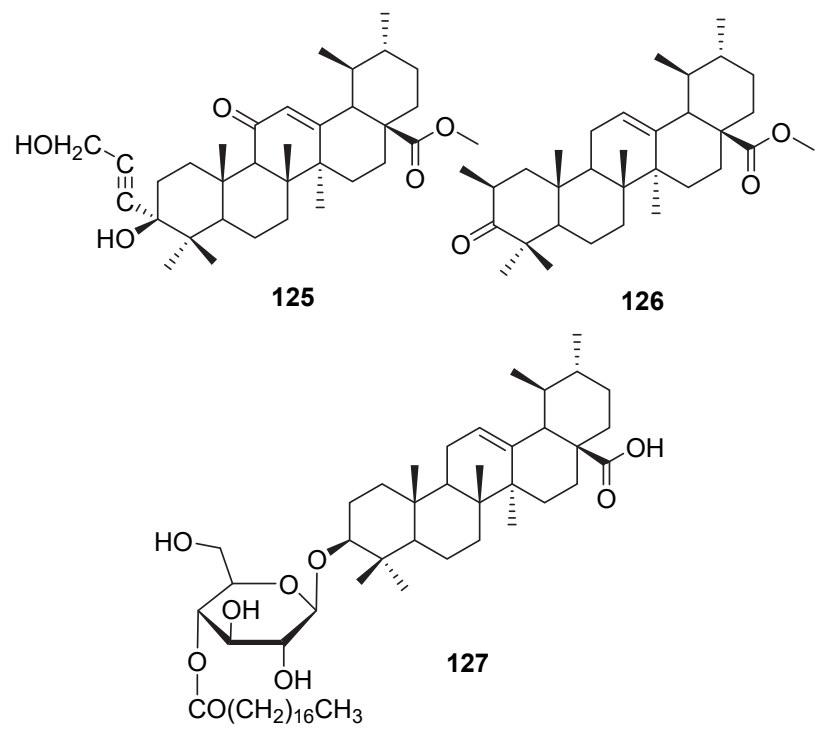

图 10 具有抗神经系统疾病活性的熊果酸衍生物的结构 Figure 10 Structures of ursolic acid derivatives for neurodegenerative diseases

\section{7 总结与展望}

随着全球气候变化, 老龄化加剧, 诸如癌症、糖尿 病、慢性呼吸道疾病和心血管疾病等患病人数逐年上升, 对人类造成致命的威胁. 临床已知治疗药物耐药性和毒 副作用的发生也日益严峻. 因此, 越来越多的科学家们 希望从自然界, 特别是从植物中寻找更好的替代药物. 药用植物中的五环三萜类化合物在治疗和预防多种非 传染性疾病中发挥关键作用. 其中熊果酸来源广泛, 含 量丰富且具有多种重要的生物学作用，包括抗炎、抗癌、 抗糖尿病、抗氧化和抗菌等作用, 但其溶解性差, 缺乏 靶标特异性等导致其生物利用度低等，限制了其临床应 用。

从上述文献综述可以看出, 科研工作者对 UA 的化 学性质及生物活性进行了大量且广泛的研究, 以进一步 提高其成药性，以期使其发挥更大的药用价值. 主要对 其 C-3(羟基)、A 环和 C-28(羧基)等进行了改造, 得到了 活性高、生物利用度好、水溶性好的多种衍生物. UA 及 其衍生物为上述非传染性疾病的治疗提供了希望，有望 在不久的将来开发出活性优异的 UA 衍生物并应用于临 床.

\section{References}

[1] Tao, Y.-B.; Xing, Y.-L.; Fang, Z.-J.; Bi, L.-W.; Zhao, Z.-D. Chem. Ind. For. Prod. 2012, 32(1), 119 (in Chinese).

(陶渊博, 邢雅丽, 方芝娟, 毕良武, 赵振东, 林产化学与工业, 2012, 32(1), 119.)

[2] Mlala, S.; Oyedeji, A. O.; Gondwe, M.; Oyedeji, O. O. Molecules 2019, 24(15), 2751.

[3] López-Hortas, L.; P, P.-L.; Gonzá Lez-MuÃ \pm Oz, M. J.; Falqué, E.; Domí-Nguez, H. Food Res. Int. 2018, 103, 130. 
[4] Khwaza, V.; Oyedeji, O. O.; Aderibigbe, B. A. Int. J. Mol. Sci. 2020, 21(16), 5920.

[5] Xiang, R.-Q.; Fan, Y. J. Pharm. Res. 2019, 38(2), 63 (in Chinese). (向润清, 范源, 药学研究, 2019, 38(2), 63.)

[6] Zhu, M. L.; Ge, W. Z.; Zhao, R. Acta Laser Biol. Sin. 2014, 23(5), 465 (in Chinese). (朱美玲，葛文中，赵芯，激光生物学报, 2014, 23(5), 465.)

[7] Wang, C.-Y.; Lin, C.-S.; Hua, C.-H.; Jou, Y.-J.; Liao, C.-R.; Chang, Y.-S.; Wan, L.; Huang, S.-H.; Hour, M.-J.; Wen, C.-L. Biomol. Ther. 2019, 27(1), 9.

[8] Xu, J.; Wang, X.-D.; Zhang, H.-Y.; Yue, J.-Y.; Zhao, Y.-Q. Nat. Prod. Res. 2018, 34(6), 1

[9] Fontana, G.; Bruno, M.; Notarbartolo, M.; Labbozzetta, M.; Poma, P.; Spinella, A.; Rosselli, S. Bioorg. Chem. 2019, 90, 103054

[10] Tu, H. Y.; Huang, A.; Wei, B. L.; Gan, K. H.; Hour, T. C.; Yang, S. C.; Pu, Y. S.; Lin, C. N. Bioorg. Med. Chem. 2009, 17(20), 7265.

[11] Gou, W.-F.; Luo, N.; Wei, H.-Q.; Wu, H.-Y.; Li, Y.-L. Pharm. Biol. 2020, 58(1), 707.

[12] Tian, T.; Liu, X.; Lee, E. S.; Sun, J.; Feng, Z.; Zhao, L.; Zhao, C. Arch. Pharmacal Res. 2017, 40(4), 1.

[13] Li, X.; Bai, X.; Wu, K.; Wang, Y. J.; Shi, W. Q.; Li, Y.; Yin, S. F. Chin. J. Org. Chem. 2012, 32(4), 703 (in Chinese). (李霞, 白雪, 吴科, 王裕军, 石万棋, 李颖, 尹述凡, 有机化学, 2012, 32(4), 703.)

[14] Mallavadhani, U. V.; Mahapatra, A.; Pattnaik, B.; Vanga, N.; Suri, N.; Saxena, A. K. Med. Chem. Res. 2013, 22(3), 1263.

[15] Liu, M. C.; Yang, S. J.; Jin, L. H.; Hu, D. Y.; Xue, W.; Song, B. A. Eur. J. Med. Chem. 2012, 58(12), 128.

[16] Liu, M. C.; Yang, S. J.; Jin, L. H.; Hu, D. Y.; Xue, W.; Yang, S. Med. Chem. Res. 2016, 25(10), 2267.

[17] Liu, H.; Li, G.-B.; Zhang, D.-L.; Liu, Y.-M.; Gao, N. J. Third Milit. Med. Univ. 2017, 39(19), 1960 (in Chinese). (刘涵, 李国兵, 张定林, 刘毅敏, 高宁, 第三军医大学学报, 2017, 39(19), 1960.)

[18] Chen, Y.-H.; Hou, X.-Y.; Zhi, D.-F.; Li, C.; Tian, T.; Sun, J.-Y.; Zhao, L.-X.; Zhao, C.-H. Chin. J. Org. Chem. 2016, 36(4), 795 (in Chinese).

(陈艳华，侯熙彦，支德福，李常，田甜，孙竞阳，赵龙铉，赵春 晖, 有机化学, 2016, 36(4), 795.)

[19] Bai, K. K.; Chen, F. L.; Guo, H. H. Chin. J. New Drug 2012, 21(22), 2667 (in Chinese).

(白锴凯, 陈芬玲, 郭养浩, 中国新药杂志, 2012, 21(22), 2667.)

[20] Cheng, W.; Dahmani, F. Z.; Zhang, J.; Xiong, H.; Wu, Y.; Yin, L.; Zhou, J.; Yao, J. Nanotechnol. 2017, 28(7), 075102.

[21] Ahn, G. Y.; Paik, D. H.; Jeong, K. Y.; Baek, S. W.; Kang, R. H.; Lee, E. S.; Choi, S. W. Macromol. Res. 2018, $26(7), 1$.

[22] Bai, K. K.; Chen, F. L.; Guo, H. H. Chin. J. New Drug 2012, 21(13), 1536 (in Chinese) (白锴凯，陈芬玲，郭养浩，中国新药杂志, 2012, 21(13), 1536.)

[23] Jiang, W.; Huang, R. Z.; Zhang, J.; Guo, T.; Zhang, M. T.; Huang, X. C.; Zhang, B.; Liao, Z. X.; Sun, J.; Wang, H. S. Bioorg. Chem. 2018, 79, 265

[24] Yu, Z.; Bai, K.-K.; Guo, Y.-H. Pharm. Biotechnol. 2010, 17(5), 382 (in Chinese). (于舟，白锴凯，郭养浩，药物生物技术, 2010, 17(5), 382.)

[25] Dong, H.-Y.; Yang, X.; Xie, J.-J.; Xiang, L.; Li, Y.-F.; Ou, M.; Chi, T.; Liu, Z.; Yu, S.-H.; Gao, Y. Biochem. Pharmacol. 2015, 93(2), 151

[26] Hua, S. X.; Huang, R. Z.; Ye, M. Y.; Pan, Y. M.; Yao, G. Y.; Zhang, Y.; Wang, H. S. Eur. J. Med. Chem. 2015, 95(10), 435.

[27] Huang, R. Z.; Hua, S. X.; Liao, Z. X.; Huang, X. C.; Wang, H. S. Med. Chem. Commun. 2017, 8(7), 1421.

[28] Wang, J.-C.; Jiang, Z.; Xiang, L.-P.; Li, Y.-F.; Ou, M.-R.; Yang, X.; Shao, J.-W.; Lu, Y.-S.; Lin, L.-F.; Chen, J.-Z. Sci. Rep. 2014, 4, 5006 .

[29] Wiemann, J.; Heller, L.; Csuk, R. Bioorg. Med. Chem. Lett. 2016, 26(3), 907.

[30] Yang, X.; Li, Y.; Jiang, W.; Ou, M.; Chen, Y.; Xu, Y.; Wu, Q.;
Zheng, Q.; Wu, F.; Wang, L.; Zou, W.; Zhang, Y. J.; Shao, J. Chem. Biol. Drug Des. 2015, 86(6), 1397.

[31] Bai, K. K.; Chen, F. L.; Zheng, Y. Q.; Guo, H. H. Chin. Pharm. J. 2012, 47(4), 265 (in Chinese). (白锴凯，陈芬玲，郑允权，郭养浩，中国药学杂志，2012，47(4), 265.)

[32] Shao, J. W.; Dai, Y. C.; Xue, J. P.; Wang, J. C.; Lin, F. P.; Guo, Y. H. Eur. J. Med. Chem. 2011, 46(7), 2652.

[33] Popov, S. A.; Kornaukhova, L. M.; Shpatov, A. V.; Grigor'ev, I. A. Chem. Nat. Compd. 2016, 52(3), 555

[34] Wolfram, R. K.; Heller, L.; Csuk, R. Eur. J. Med. Chem. 2018, 152, 21.

[35] Rashid, S.; Dar, B. A.; Majeed, R.; Hamid, A.; Bhat, B. A. Eur. J. Med. Chem. 2013, 66(66C), 238.

[36] Yu, T.-T.; Li, L.; Cui, H.-B.; Meng, Y.-Q. Chem 2017, 80(11), 74.

[37] Yan-Qiu, M.; Dan, L.; Zhong-Wei, B.; Ling-Li, C.; Hong-Ru, A. Acta Pharm. Sin. 2011, 46(5), 556 (in Chinese). (孟艳秋，刘丹，白忠伟，蔡伶俐，艾宏儒，药学学报，2011， 46(5), 556.)

[38] Meng, Y.-Q.; Song, Y.-L.; Yan, Z.-K.; Xia, Y. Molecules 2010, 15(6), 4033

[39] Xu, C.-D.; Meng, Y.-Q. J. Shenyang Univ. Chem. Technol. 2020, 34(1), 18 (in Chinese). (徐川东, 孟艳秋, 沈阳化工大学学报, 2020, 34(1), 18.)

[40] Meng, Y.-Q.; Xu, C.-D.; Yu, T.-T.; Li, W.; Li, Q.-W.; Li, X.-X. J. Asian Nat. Prod. Res. 2019, 24(4), 359.

[41] Meng, Y.-Q.; Ding, Y.; Yang, Z.; Zhang, M. Drugs Clin. 2014, 29(2), 116 (in Chinese). (孟艳秋, 丁一, 张萌, 杨哲, 现代药物与临床, 2014, 29(2), 116.)

[42] Yao, Y.; Zhang, X.; Wang, Z.; Zheng, C.; Li, P.; Huang, C.; Tao, W.; Xiao, W.; Wang, Y.; Huang, L.; Yang, L. J. Ethnopharmacol. 2013 , $150(2), 619$.

[43] Zhang, L.-F.; Meng, Y.-Q. J. Shenyang Univ. Chem. Technol. 2016, 30(2), 117 (in Chinese). (张良锋, 孟艳秋, 沈阳化工大学学报, 2016, 30(2), 117.)

[44] Li, A. L.; Hao, Y.; Wang, W. Y.; Liu, Q. S.; Gu, W. Int. J. Mol. Sci. 2020, 21(8), 2876.

[45] Jin, X. Y.; Chen, H.; Li, D. D.; Li, A. L.; Gu, W. J. Enzyme Inhib. Med. Chem. 2019, 34(1), 955.

[46] Liu, X.-Y.; Gao, X.-Q.; Jin, X.-J.; Zhao, C.-H.; Feng, Z.-H.; Sui, Y.; Zhao, L.-X.; Yan, X. Chin. J. Org. Chem. 2018, 38(12), 99 (in Chinese).

(刘新宇, 高雪琴, 金学军, 赵春晖, 冯中华, 隋悦, 赵龙铉, 阎 欣, 有机化学, 2018, 38(12), 99.)

[47] Zhang, T.; He, B.-E.; Yuan, H.; Feng, G.-L.; Chen, F.-L.; Wu, A.-Z.; Zhang, L.-L.; Lin, H.-R.; Zhuo, Z.-J.; Wang, T. Chem. Biodiversity 2019, 16(6), e1900111.

[48] Wu, J.; Zhang, Z. H.; Zhang, L. H.; Jin, X. J.; Ma, J.; Piao, H. R. Bioorg. Med. Chem. Lett. 2019, 29(6), 853.

[49] Fan, H.-T.; Geng, L.; Yang, F.; Dong, X.; He, D.; Zhang, Y. Eur. J. Med. Chem. 2019, 176, 61.

[50] Pei, T.; Wang, Y.-Y. J. Chin. Pharm. Univ. 2017, 48(1), 31 (in Chinese). (裴婷, 王玉英，中国药科大学学报, 2017, 48(1), 31.)

[51] Chen, Y.; Li, C.; Zheng, Y.; Gao, Y.; Hu, J.; Chen, H. Bioorg. Med. Chem. Lett. 2017, 27(4), 1007

[52] Li, W.; Zhang, H.; Nie, M.; Wang, W.; Liu, Z.; Chen, C.; Chen, H.; Liu, R.; Baloch, Z.; Ma, K. Oncol. Lett. 2018, 15(2), 2323.

[53] Dar, B. A.; Lone, A. M.; Shah, W. A.; Qurishi, M. A. Eur. J. Med. Chem. 2016, 111, 26.

[54] Finlay, H. J.; Honda, T.; Gribble, G. W.; Danielpour, D.; Benoit, N. E.; Suh, N.; Williams, C.; Sporn, M. B. Bioorg. Med. Chem. Lett. 1997, 7(13), 1769

[55] Gu, W.; Jin, X. Y.; Li, D. D.; Wang, S. F.; Tao, X. B.; Chen, H. Bioorg. Med. Chem. Lett. 2017, 27(17), 4128.

[56] Gu, W.; Hao, Y.; Zhang, G.; Wang, S. F.; Miao, T. T.; Zhang, K. P. Bioorg. Med. Chem. Lett. 2015, 25(3), 554

[57] Lin, R. X.; Gong, L. L.; Fan, L. M.; Zhao, Z. K.; Yang, S. L. Int. J. 
Clin. Exp. Pathol. 2015, 8(2), 1427.

[58] Mendes, V. I. S.; Bartholomeusz, G. A.; Ayres, M.; Gandhi, V.; Salvador, J. A. R. Eur. J. Med. Chem. 2016, 123, 317.

[59] Sun, L.; Li, B.; Su, X.; Chen, G.; Li, Y.; Yu, L.; Li, L.; Wei, W. J. Med. Chem. 2017, 60(15), 6638

[60] Jin, X.-Y.; Chen, H.; Tao, X.-B.; Wang, S.; Gu, W. J. For. Eng 2018, (1), 54

[61] Pan, H.-S.; Li, L.; Cui, H.-B.; Yang, L.-N.; Yu, T.-T.; Meng, Y.-Q. Acta Pharm. Sin. 2017, 52(12), 1890 (in Chinese). (潘洪双, 李否, 崔华博, 杨丽娜, 于婷婷, 孟艳秋, 药学学报, 2017, 52(12), 1890.)

[62] Meng, Y.-Q.; Cao, J.; Tang, Y.; Lu, X.-Y.; Liu, L.-W. Chin. J. Org Chem. 2016, 36(5), 1080 (in Chinese). (孟艳秋, 曹佳, 汤义，鹿学宇，刘立伟，有机化学，2016，36(5), 1080.)

[63] Wang, Y.-Y.; Xiong, H. Strait. Pharm. J. 2017, 29(2), 268 (in Chinese) (王玉英, 熊慧, 海峡药学, 2017, 29(2), 268.)

[64] Wang, Y.-Y.; Xiong, H. Strait. Pharm. J. 2016, 28(12), 273 (in Chinese).

(王玉英, 熊慧, 海峡药学, 2016, 28(12), 273.)

[65] Wei, Z.; Di, H.; Zhou, Y.; Zhang, Y.; Qiang, S.; Li, J. Y.; Hu, L. H.; Jia, L. Biochim. Biophys. Acta. Gen. Subj. 2006, 1760(10), 1505.

[66] Guzmán-Ávila, R.; Flores-Morales, V.; Paoli, P.; Camici, G.; Ramírez-Espinosa, J. J.; Cerón-Romero, L.; Navarrete-Vázquez, G.; Hidalgo-Figueroa, S.; Yolanda, R. M.; Villalobos-Molina, R. Drug Dev. Res. 2018, 79(2). 70.

[67] Kazmi, I.; Rahman, M.; Afzal, M.; Gupta, G.; Saleem, S.; Afzal, O.; Shaharyar, M. A.; Nautiyal, U.; Ahmed, S.; Anwar, F. Fitoterapia 2012, 83(1), 142.

[68] Wu, P. P.; Zhang, K.; Lu, Y. J.; He, P.; Zhao, S. Q. Eur. J. Med. Chem. 2014, 80, 502 .

[69] Wu, P. P.; Zhang, B. J.; Cui, X. P.; Yang, Y.; Jiang, Z. Y.; Zhou, Z. H.; Zhong, Y. Y.; Mai, Y. Y.; Ouyang, Z.; Chen, H. S. Sci. Rep. 2017, 7, 45578.

[70] Gao, Y.; Li, Z.; Xie, X.; Wang, C.; You, J.; Mo, F.; Jin, B.; Chen, J.; Shao, J.; Chen, H.; Jia, L. Eur. J. Pharm. Sci. 2015, 70, 55.

[71] Chen, L.; Yang, X. S.; Yang, X.; Ye, L. H.; Hao, X. J. J. Chin. Pharm. Univer. 2010, 41(3), 222 (in Chinese). (陈磊, 杨小生, 杨娟, 叶林虎, 郝小江, 中国药科大学学报, 2010, 41(3), 222.)

[72] Zhang, L.; Dong, J.; Liu, J.; Zhang, L.; Kong, L.; Yao, H.; Sun, H. Med. Chem. 2013, $9(1), 118$.

[73] Chen, C.; Sun, R.; Sun, Y.; Chen, X.; Chen, D. Bioorg. Med. Chem. Lett. 2019, 30(2), 126824.

[74] Wei, Z. Y.; Chi, K. Q.; Wang, K. S.; Wu, J.; Liu, L. P.; Piao, H. R. Bioorg. Med. Chem. Lett. 2018, $28(10), 1797$.

[75] Nelson, A. T.; Camelio, A. M.; Claussen, K. R.; Cho, J.; Tremmel, L.; Digiovanni, J.; Siegel, D. Bioorg. Med. Chem. Lett. 2015, 25(19), 4342.

[76] Zhang, C.; Xu, S. H.; Ma, B. L.; Wang, W. W.; Yu, B. Y.; Zhang, J. Bioorg. Med. Chem. Lett. 2017, 27(11), 2575.

[77] Li, C. H.; Yang, C. L.; Zhang, K.; Shi, W. Q.; Li, J. Z.; Li, Y.; Yin, S. F. Chin. J. Org. Chem. 2012, 32 (1), 133 (in Chinese). (李财虎, 杨聪玲, 张宽, 石万棋, 李剑忠, 李颖, 尹述凡, 有机 化学, 2012, 32(1), 133.)

[78] Tan, J.; Huang, W.; Chen, S.-L.; Yue, Y. Acta Pharm. Sin. 2016, 51(6), 938 (in Chinese) (谭娟, 黄微, 陈善龙, 岳源, 药学学报, 2016, 51(6), 938.)

[79] Li, C.; Chen, J.; Yuan, W.; Zhang, W.; Chen, H.; Tan, H. Iubmb. Life 2020, 72(4), 632

[80] Fu, H. J.; Zhou, Y. R.; Bao, B. H.; Jia, M. X.; Zhao, Y.; Zhang, L.;
Li, J. X.; He, H. L.; Zhou, X. M. J. Med. Chem. 2014, 57(11), 4692.

[81] Fu, H. J.; Zhao, Y.; Zhou, Y. R.; Bao, B. H.; Du, Y.; Li, J. X. Eur. J. Pharm. Sci. 2015, 76, 33

[82] Yu, S. G.; Zhang, C. J.; Xu, X. E.; Sun, J. H.; Zhang, L.; Yu, P. F. Int. J. Clin. Exp. Pathol. 2014, 8(4), 3681.

[83] Kong, L.-B.; Li, S.-S.; Liao, Q.-J.; Zhang, Y.-N.; Sun, R.-N.; Zhu, X.-D.; Zhang, Q.-H.; Wang, J.; Wu, X.-Y.; Fang, X.-N. Antiviral Res. 2013, 98(1), 44.

[84] Zhong, Y.; Dai, Z.; Xu, Y.; Teng, Y.; Wu, B. Eur. J. Pharm. Sci. 2012, 45(1-2), 110

[85] Li, S.-M.; Jia, X.-H.; Li, H.; Ye, Y.-L.; Song, G.-P. Bioorg. Med. Chem. Lett. 2020, 30(22), 127518.

[86] Song, G.; Shen, X.; Li, S.; Li, Y.; Liu, Y.; Zheng, Y.; Lin, R.; Fan, J.; Ye, H.; Liu, S. Eur. J. Med. Chem. 2015, 93, 431.

[87] Luan, T.; Jin, C. M.; Gong, G. H.; Quan, Z. S. J. Enzyme Inhib. Med. Chem. 2019, 34(1), 761.

[88] Sifaoui, I.; Rodríguez-Expósito, R. L.; Reyes-Batlle, M.; RizoLiendo, A.; Piero, J. E.; Bazzocchi, I. L.; Lorenzo-Morales, J.; Jiménez, I. A. Pathogens 2019, 8(3), 130.

[89] Wang, P. Y.; Xiang, M.; Luo, M.; Liu, H. W.; Zhou, X.; Wu, Z. B.; Liu, L. W.; Li, Z.; Yang, S. Pest. Manage. Sci. 2020, 76(8), 2746.

[90] Cunha, L. C. S.; Silva, M. L. a. E.; Furtado, N. a. X. C.; Vinholis, A. H. C.; Martins, C. H. G.; Filho, A. A. D. S.; Cunha, W. R. Z. Naturforsch., C: J. Biosci. 2007, 62(9 10), 668.

[91] Do Nascimento, P. G. G.; Lemos, T. L. G.; Bizerra, A. M. C.; Arriaga, Â. M. C.; Ferreira, D. A.; Santiago, G. M. P.; Braz-Filho, R.; Costa, J. G. M. Molecules 2014, $19(1), 1317$.

[92] Dwivedi, G. R.; Maurya, A.; Yadav, D. K.; Khan, F.; Darokar, M. P.; Srivastava, S. K. Chem. Biol. Drug Des. 2015, 86(3), 272.

[93] Jabeen, M.; Ahmad, S.; Shahid, K.; Sadiq, A.; Rashid, U. Front. Chem. 2018, 6, 55 .

[94] Huang, L. R.; Luo, H.; Yang, X. S.; Lei, C.; Zhang, J. X.; Wang, D. P.; Hao, X. J. Med. Chem. Res. 2014, 23(11), 4631.

[95] Meng, Y.-C.; Zhan, J.-H.; Xiao, S.-P.; Tan, Y.; Liao, M.-F.; Zhang, Y.-L.; Li, L.; Pei, G. J. Hunan Univ. Chin. Med. 2017, 37(5), 493 (in Chinese).

(孟英才, 詹济华, 肖水平, 谭洋, 廖美芳, 张雨林, 李玲, 裴刚, 湖南中医药大学学报, 2017, 37(5), 493.)

[96] Gnoatto, S.; Dalla-Vechia, L., Cl; Dassonville-Klimpt, A.; Da-Nascimento, S.; Mossalayi, D.; Guillon, J.; Gosmann, G.; Sonnet, P. J. Enzyme Inhib. Med. Chem. 2008, 23(5), 604.

[97] Innocente, A. M.; Silva, G. N. S.; Laura Nogueira, C.; Moraes, M. S.; Myna, N.; Pascal, S.; Grace, G.; Garcia, C. R. S.; Gnoatto, S. C. B. Molecules 2012, 17(10), 12003

[98] Silva, G. N. D.; Maria, N. R.; Schuck, D. C.; Cruz, L. N.; Moraes, M. S. D.; Nakabashi, M.; Graebin, C.; Gosmann, G.; Garcia, C. R.; Gnoatto, S. C. Malar. J. 2013, 12(1), 89.

[99] Cargnin, S. T.; Staudt, A. F.; Medeiros, P.; Sol, D. D. M. S.; Santos, A. P. D. a. D.; Zanchi, F. B.; Gosmann, G.; Puyet, A.; Teles, C. B. G.; Gnoatto, S. B. Bioorg. Med. Chem. Lett. 2018, $28(3), 265$.

[100] Bitencourt, F. G.; Vieira, P. D. B.; Meirelles, L. C.; Rigo, G. V.; Silva, E. F. D.; Gnoatto, S. C. B.; Tasca, T. Parasitol. Res. 2018 , $117(5), 1$.

[101] Loesche, A.; Köwitsch, A.; Lucas, S. D.; Al-Halabi, Z.; Sippl, W. Al-Harrasi, A; Csuk, R. Bioorg. Chem. 2019, 85, 23.

[102] Imran, K.; Muhammad, A.; Gaurav, G.; Firoz, A. CNS Neurosci. Ther. 2012, 18(9), 799.

[103] Kazmi, I.; Gupta, G.; Afzal, M.; Anwar, F. Asian Pac. J. Trop. Dis. 2012, 2(Suppl. 1), S453.

[104] Imran, K.; Gaurav, G.; Muhammad, A.; Mahfoozur, R.; Firoz, A. CNS Neurosci. Ther. 2012, 18 (8), 707. 